\title{
Dissecting Mutational Allosteric Effects in Alkaline Phosphatases Associated with Different Hypophosphatasia Phenotypes: An Integrative Computational Investigation
}

Fei Xiao ${ }^{1 \pi}$, Ziyun Zhou ${ }^{1 \pi}$, Xingyu Song ${ }^{2}$, Mi Gan ${ }^{1}$, Jie Long $^{1}$, Gennady M Verkhivker ${ }^{3,4}$, Guang $\mathrm{Hu}^{1 *}$

${ }^{1}$ Center for Systems Biology, Department of Bioinformatics, School of Biology and Basic Medical Sciences, Soochow University, Suzhou 215123, China

${ }^{2}$ Department of Chemistry, Institutes of Biomedical Sciences, Fudan University, Shanghai 200438, China

${ }^{3}$ Department of Computational and Data Sciences, Chapman University, One University

Drive, Orange, California 92866, USA

${ }^{4}$ Department of Biomedical and Pharmaceutical Sciences, Chapman University Pharmacy

School 9401 Jeronimo Rd, Irvine, CA 92618, USA

* Corresponding author

Email: huguang@suda.edu.cn (GH)

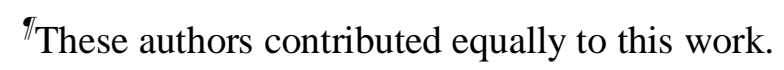




\section{Abstract}

Hypophosphatasia (HPP) is a rare inherited disorder characterized by defective bone mineralization, is highly variable in its clinical phenotype. The disease occurs due to various loss-of-function mutations in $A L P L$, the gene encoding tissue-nonspecific alkaline phosphatase (TNSALP). In this work, a data-driven and biophysics-based approach for large-scale analysis of $A L P L$ mutations - from nonpathogenic to severe HPPs is proposed. Allosteric molecular signatures of $A L P L$ mutations were determined using an integrated pipeline of synergistic approaches including sequence and energetic-based analysis, structural topology network modeling and elastic network models. Statistical analysis of molecular features computed for the $A L P L$ mutations showed a significant difference between the control and mild and severe HPP phenotypes, and the developed machine learning model suggested that the topological network parameters could serve as is a robust indicator for severe mutations. Molecular dynamics simulations coupled with protein structure network was employed to analyze the effect of single-residue variation on conformational dynamics of TNSALP dimers, and we found that severe disease-associated mutations have a significantly greater effect on allosteric communications. The results of this study suggested that $A L P L$ mutations associated with mild and severe HPPs tend to have different effects on protein stability on local scale and long-range communications caused by network rewiring. By linking disease phenotypes with allosteric molecular signatures, the proposed integrative computational approach elucidates the complex sequence-structure-allostery relationships of $A L P L$ mutations and dissects the role of allosteric effects in the pathogenesis of HPPs. 


\section{Author Summary}

The understanding of mutational genotype- disease phenotype relationship is a fundamental step for enabling precision medicine. It remains a challenging task to assess the molecular principle of the genotype- phenotype relationship. By focusing on Hypophosphatasia, a rare inherited disorder, as an example, we performed comprehensive analysis of single-amino-acid mutations in the encoded protein of Tissue Nonspecific Alkaline Phosphatase associated in terms of their sequence, structure, and dynamics properties. We further developed a machine learning -based method to classify different disease phenotypes, and the interpretability of the classification model was addressed by the structural-functional analysis of network topological important mutations. Our results highlighted the allosteric propensity of severe mutations, and show the potential allosteric principle of genotype- phenotype relationship.

\section{Introduction}

Hypophosphatasia (HPP) is a rare autosomal dominant or recessive metabolic disorder, first described in 1948 by Rathbun,[1] which constitutes a complex, multisystemic disease.[2] The clinical presentation is highly variable, depending on the type of mutation and the inheritance mechanism. From a prenatal lethal form with no skeletal mineralization to a mild form with late adult onset, HPP can be classified into six subtypes, including perinatal, infantile, prenatal benign, childhood, adult and odonto.[3] In general, perinatal and infantile subtypes represent severe forms of HPP, while childhood, adult, odonto, and prenatal benign constitute mild phenotypes.[4] HPP is caused by loss-of-function mutations in the ALPL gene encoding the

Tissue Nonspecific Alkaline Phosphatase (TNSALP).[5] TNSALP is a membrane-bound 
metalloenzyme, whose activity is reduced by various mutations in the $A L P L$ gene, leading to increased inorganic pyrophosphate, which in turn cause different HPP phenotypes.[6] As an enzyme replacement therapy, ENB-0040 is a bone-targeted, recombinant human TNSALP that prevents the manifestations of HPP.[7] However, to date, there is no established treatment for HPPs,[8] due to the little knowledge about the relationship between mutations in the gene responsible for HPPs and phenotypic variability.

The ALPL gene is localized on chromosome 1p36.1-34 and consists of 12 exons distributed over $50 \mathrm{~kb} .[9,10] A L P L$ mutation detection is important for recurrence risk assessment and prenatal diagnosis.[11] To date, more than 400 ALPL gene mutations have been reported worldwide and about $80 \%$ of these mutations are missense. Although HPP is caused by homozygous, heterozygous, or compound heterozygous $A L P L$ mutations,[12] most of these mutations cause change in a single amino acid in TNSALP. Mutations in ALPL would reduce the enzyme activity of TNSALP mutant proteins to a varying degree, with residual activities often exhibiting different enzymatic properties from wide-type TNSALP. By measuring the effects of amino acid changes on TNSALP dimer stability, the relationship between $A L P L$ missense variants and TNSALP enzymatic activity can be predicted.[13] Despite recent progress, the correlation between these mutations and the six HPP subtypes is not well established, and thus the molecular mechanisms underlying the genotypic-phenotypic relationships of HPP remain unclear.[14] A more detailed molecular characterization of $A L P L$ mutations can help to establish the etiology, pathogenesis, and onset of HPP. Moreover, understanding molecular signatures of pathogenic mutations may provide a better strategy for inhibiting TNSALP and tools to govern rationale molecular targeted strategy.[15] 
TNSALP is a homodimeric protein that contains several known domains, including five principal functional domains: a catalytic site, calcium-binding site, crown domain, homodimer interface, and N-terminal alpha helix.[12] The assignment of various mutations to the five functional domains of the TNSALP structure model has contributed to the current understanding of the genotypic and phenotypic interrelationship of HPP.[16] For example, most of the severe missense mutations were localized in crucial domains, such as the active site, the vicinity of the active site, and the homodimer interface. The structural importance of the crown domain has also been highlighted, with respect to the catalytic function of TNSALP.[17] In addition, there is structural evidence to support the concept that these crucial domains are also involved in allosteric properties of TNSALP, since the catalytic activity depends on its homodimeric configuration from which the dominant negative effect of some loss-of-function ALPL mutations is derived. Although mammalian alkaline phosphatases have been known as allosteric enzymes for many years,[18] the dynamics-driven allosteric signaling pathways is yet to be investigated at an atomistic level. Therefore, the elucidation of the conformational dynamics and allosteric signatures of TNSALP caused by ALPL mutations would provide greater insight into genotype-phenotype relationship in HPP.[19, 20]

In order to predict catalytic efficiency[21] and potential pathogenicity of mutations,[22, 23] different levels of computational methods, from sequence analysis to structural and dynamics analyses have been developed. From a molecular evolution perspective, sequence-based methods have shown that pathogenic mutations always involve conserved amino acids.[24] Furthermore, it has been pointed out that disease-causing mutations frequently involve a drastic change in amino acid physicochemical properties, such as charge, 
hydrophobicity, and geometry, and are fewer surface exposed than polymorphic mutations.[25] Structural modeling combined with network theory has been widely exploited in studying protein topology and dynamics.[26, 27] The description of protein structure as a structural network provides an efficient tool to dissect complex protein functions.[28] Based on protein structural network model, different topological parameters and algorithms have been developed to describe structural changes associated with mutations.[29, 30] Conformational dynamics would provide more insight into the physical basis of the effect of missense variants.[31] Elastic network models (ENMs) have long been established in the analysis of protein dynamics in the context of normal model analysis,[32, 33] and have now become an extremely useful approach to predict the effects of single-point mutations on protein stability.[34] The calculation has revealed that mutations are significantly enriched on hinge-neighboring residues in oncogenes and tumor suppressor genes.[35] By incorporating dynamics descriptors based on ENM with sequence and structure-dependent properties, the prediction accuracy of impact of variants on biological function has greatly increased.[36] Machine learning models that integrate sequence, structure, and ensemble-based features have been developed to classify mutation types.[37-39] Furthermore, biophysical simulations combined with structure-based modeling of residue interaction networks have also been used to reveal the functional role of mutation hotspots in molecular communication in some tumor suppressor proteins,[40] regulatory complexes including HSp90[41], and SARS-CoV-2 Spike Protein,[42] as well as classify PTEN missense variants corresponding to cancer or autism spectrum disorder.[43-45]

Our recent works have highlighted that biophysics-based and data-driven approaches, 
including genomic analysis, coevolution and network-based modeling provide an array of powerful tools to study disease mutations in genomic medicine and allosteric interactions.[46-49] In this work, based on this concept, we propose integrated computational approach for the large-scale analysis of disease mutations. As shown in Fig 1, we collected diverse $A L P L$ single-point mutations associated with neutral, mild, and severe phenotypes from various scientific gene mutation databases; the computational pipeline consisted of three steps. First, by performing three levels of analysis, including conservation and co-evolution analysis based on sequence level, structural modeling, energetic analysis and protein structure network (PSN) modeling based on structural level, and ENM-related calculation based on dynamics level, different molecular and network signatures were attributed to three types of mutations. Second, statistical analysis including creation of a random forest mode, was performed to test which molecular signatures can serve as robust predictors for classifying the three kinds of mutations. Finally, through integration of long-range perturbation dynamics and network-based approaches, we have quantified allosteric potential of selected mutation residues. Our study characterizes the mutational landscape of $A L P L$ through modeling and analysis of molecular signatures and allosteric effects of mutations,[50] providing new insights into the genotype-phenotype interrelationship in HPP.[51] 


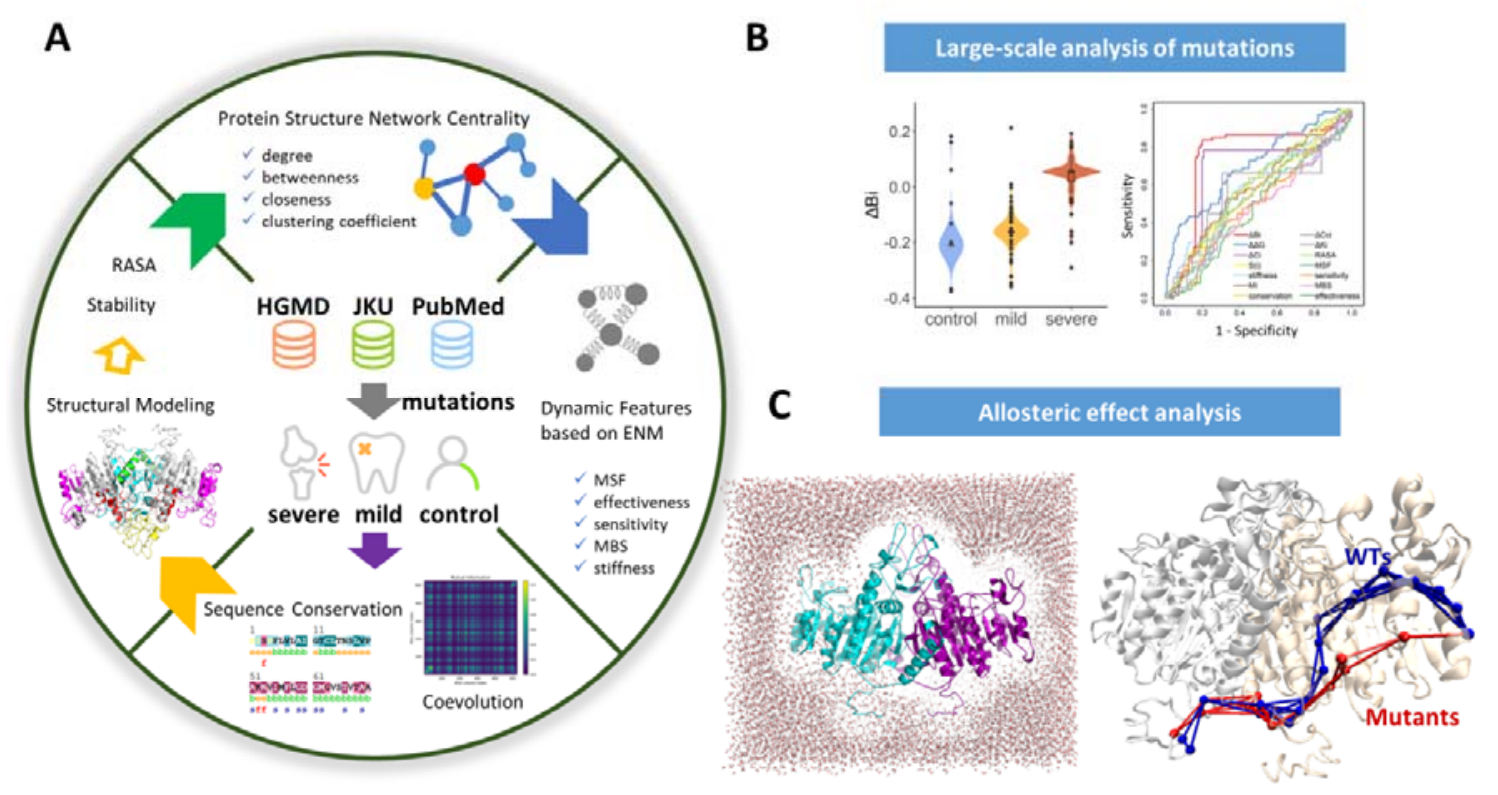

Fig 1. The computational workflow. (A) Beginning with the collection of $A L P L$ mutations associated with different HPP phenotypes and the computational modeling of TNSALP protein structure, molecular signatures of mutational hotspots were calculated. In addition to normally used signatures, three levels of parameters for describing mutations were analyzed: conservation and co-evolution analysis at sequence level, PSN-based network matrices at structural level, ENM-related features at dynamics level. (B) The classification and prediction of pathogenicity of $A L P L$ mutations based on the statistical analysis of molecular signatures and the construction of machine learning models. (C) The allosteric effect analysis of predicted mutations by single-residue perturbation, molecular dynamics and long-range pathway analysis.

\section{Materials and Methods}

\section{Data Collection of ALPL Mutations}


With the goal of classifying various $A L P L$ variants according to different phenotypes, 242 single-point loss of function variants were selected in HPP patients with mild and severe phenotypes and the control group from the $A L P L$ gene mutation database (https://alplmutationdatabase.jku.at, accessed on 11 January 2021), the Human Gene Mutation Database (http://www.hgmd.cf.ac.uk/ac/index.php), the Locus Specific Mutation Databases (http://www.hgmd.cf.ac.uk/docs/oth_mut.html), and literature in the PubMed database. The collated data set of ALPL mutations associated with mild and, severe HPP phenotypes and putative functionally nonsense variants, as well as their molecular signatures, are listed in S1 Table.

\section{Sequence Conservation and Coevolution Analyses}

By using the Consurf server,[52] the conservation scale for each residue in the TNSALP protein was calculated (range from 1 through 9, where 1 denotes the node least conserved and 9 denotes the most conserved sites). The refined multiple sequence alignment (MSA) of the TNSALP protein can also be retrieved from the Consurf server, which has been subjected to Shannon information entropy $\boldsymbol{S}(i)$ and mutual information $(\boldsymbol{M I})$ calculations. $S(i)$ measures the variability of specific sites of protein sequences, which is calculated as

$$
S(i)=-\sum_{a i=1}^{20} P(a i) \log P(a i)
$$

where $P(a i)$ is the probability of occurrence of amino acid type at the $i^{\text {th }}$ column. $S(i)$ varies in the range $0 \leq S(i) \leq 3.0$, a lower $S(i)$ implies higher evolutionary conservation. Similarly, $M I$ was applied as a measure of the degree of intra-molecular co-evolution between residues. The $M I$ associated with the $\mathrm{i}^{\text {th }}$ and $\mathrm{j}^{\text {th }}$ sequence positions is defined as an $N \times N$ 
matrix of the form

$$
I(i, j)=\sum_{x i=1}^{21} \sum_{y j=1}^{21} P(x i, y j) \log \frac{\mathrm{P}(\mathrm{xi}, \mathrm{yj})}{\mathrm{P}(\mathrm{xi}) \mathrm{P}(\mathrm{yj})}
$$

where $\mathrm{P}(\mathrm{xi}, \mathrm{yj})$ is the joint probability of observing amino acid types $x$ and $y$ at the respective sequence positions, $i$ and $j ; \mathrm{P}(\mathrm{xi})$ is the marginal/singlet probability of amino acid of type $x$ at the $i^{\text {th }}$ position. $I(i, j)$ varies in the range $\left[0, I_{\max }\right]$, corresponding to fully uncorrelated and most correlated pairs of residues. The co-evolution of a mutation was measured by the average $M I$ values corresponding to each residue. The calculation of $S(i)$ and $M I$ were performed by Evol.[53]

\section{Structural Modeling and Protein Stability Analysis}

A protein homology model for human TNSALP was constructed by using the MODELLER V9.19[54] platform, using a template corresponding to the human placental alkaline phosphatase (PDB id: 1ZED[55]). The template had a sequence identity of 57\% to TNSALP and an $\mathrm{X} \square$ ray crystal structure resolution of $1.57 \AA$. Each single $A L P L$ missense variant and its mutant structures were automatically generated by FoldX,[56] and its effect on protein stability can be measured by the difference folding Gibbs free energy ( $\Delta \Delta G$ values) between the wild type (WT) and the mutated forms of TNSALPs. The accessible surface area (ASA) is the atomic surface area of a protein that is accessible to a solvent, and the relative ASA $(\boldsymbol{R A S A})$ attribute is the per-residue ratio between the calculated ASA and 'standard' ASA for a particular mutational residue. As a measure of amino acid side-chain accessibility, the RASA also serves a quantitative predictor of variants' pathogenicity.[57] The RASA calculation of TNSALP is done through PSAIA 1.0.[58] 


\section{Protein Structure Network Analysis}

Protein structure networks for the TNSALPs were constructed and analyzed by using the NACEN R package.[59] A node in the network denotes a single amino acid residue, and edges are defined by the environment-dependent residue contact energy between two nodes.[60] Based on PSNs, some network centralities have been defined.[61] The simplest centrality measure is the degree $\left(\boldsymbol{K}_{i}\right)$ of a node $i$ in PSNs, defined as the total number of nodes that it is directly connected to. The betweenness centrality $\boldsymbol{B}_{i}$ was defined as the number of times residue $i$ was included in the shortest path between each pair of residues in the protein, normalized by the total number of pairs. It is calculated by

$$
\mathrm{B}_{\mathrm{i}}=\sum_{\mathrm{j}, \mathrm{k} \in \mathrm{N}, \mathrm{j} \neq \mathrm{k}} \frac{\mathrm{n}_{\mathrm{k} k}(\mathrm{i})}{\mathrm{n}_{\mathrm{jk}}}
$$

where $\mathrm{n}_{\mathrm{jk}}$ is the number of shortest paths connecting $j$ and $k$, while $\mathrm{n}_{\mathrm{jk}}(\mathrm{i})$ is the number of shortest paths connecting $j$ and $k$ and passing through $i$. The closeness centrality $\boldsymbol{C}_{i}$ for a node was calculated by the reciprocal of the average shortest path length, which can be calculated as follows:

$$
C_{i}=\frac{(n-1)}{\sum_{k \in N, k \neq m} L(m, k)}
$$

where $\mathrm{N}$ is the set of all modes and $n$ is the number of nodes in the network. The clustering coefficient $\left(\boldsymbol{C} \boldsymbol{c}_{i}\right)$ measures the degree to which nodes tend to cluster together and is defined as:

$$
\mathrm{Cc}_{\mathrm{i}}=\frac{2 \mathrm{e}_{\mathrm{i}}}{\mathrm{K}_{\mathrm{i}}\left(\mathrm{K}_{\mathrm{i}}-1\right)}
$$

where $K_{i}$ is the degree of node $i$ and $e_{i}$ is the number of connected pairs between all neighbors of $i$. 


\section{Dynamic Features based on Elastic Network Models}

In ENMs,[32, 33] each node represents a $\mathrm{C} \alpha$ atom in proteins and each edge is a spring $\gamma$ for connecting two sites within a given cutoff distance $r_{c}$. Two most commonly used ENM methods, the GNM and the ANM, are adapted in this paper. The total potential energy of the ANM and GNM systems with $\mathrm{N}$ nodes are expressed as

$$
\begin{array}{r}
\mathrm{V}_{\mathrm{GNM}}=-\frac{\gamma}{2}\left[\sum_{\mathrm{i}=1}^{\mathrm{N}-1} \sum_{\mathrm{j}=\mathrm{i}+1}^{\mathrm{N}}\left(R_{i j}-R_{i j}^{0}\right) \cdot\left(R_{i j}-R_{i j}^{0}\right) \Gamma_{i j}\right] \\
\mathrm{V}_{\mathrm{ANM}}=-\frac{\gamma}{2}\left[\sum_{\mathrm{i}=1}^{\mathrm{N}-1} \sum_{\mathrm{j}=\mathrm{i}+1}^{\mathrm{N}}\left(R_{i j}-R_{i j}^{0}\right)^{2} \Gamma_{i j}\right]
\end{array}
$$

where $\mathrm{R}_{\mathrm{ij}}$ and $\mathrm{R}_{\mathrm{ij}}^{0}$ are the instantaneous and equilibrium distances between nodes $i$ and $j$, and $\Gamma_{i j}$ is the $i j^{\text {th }}$ element of the $\mathrm{N} \times \mathrm{N}$ Kirchoff matrix $\Gamma$, which is written as

$$
\Gamma_{i j}=\left\{\begin{array}{cr}
-1 & i \neq j, R_{i j} \leq r_{c} \\
0 & i \neq j, R_{i j}>r_{c} \\
-\sum_{i, i \neq j} \Gamma_{i j} & i=j
\end{array}\right.
$$

In our study, $\mathrm{r}_{\mathrm{c}}$ between protein nodes are $7 \AA$ and $13 \AA$ for GNM and ANM, respectively. In comparison with GNM that only measure fluctuation, ANM provides additional information of motion directions of each residue. Herein, the GNM and ANM calculations were performed by ProDy.[62]

The normal modes are extracted by eigenvalue decomposition $\Gamma=U \wedge U^{T}$. $U$ is the orthogonal matrix whose $\mathrm{k}^{\text {th }}$ column $\mathrm{U}_{\mathrm{k}}$ is the $\mathrm{k}^{\text {th }}$ mode eigenvector, and $\wedge$ is the diagonal matrix of eigenvalues, $\lambda_{\mathrm{k}}$. Mean-square fluctuations $(\boldsymbol{M S F})$ of a residue are given by

$$
\left\langle\left(\Delta \mathrm{R}_{\mathrm{i}}\right)^{2}\right\rangle=\frac{3 \mathrm{k}_{\mathrm{B}}^{\mathrm{T}}}{\gamma} \sum_{\mathrm{k}}\left[\left(\mathrm{U}_{\mathrm{k}} \Lambda \mathrm{U}_{\mathrm{k}}^{\mathrm{T}}\right)^{-1}\right]_{\mathrm{ii}}
$$

where $\mathrm{k}_{\mathrm{B}}$ and $\mathrm{T}$ represent Boltzmann constant and temperature, respectively. Based on ENM calculation, several other kinds of matrixes can be generated. Form Perturbation Response Scanning (PRS) matrix,[63] two dynamic features of effectiveness and sensitivity 
were defined as row and column averages of the matrix, respectively. The effector residues most effectively propagate signals in response to external perturbations. The sensor residues can easily sense signals and respond with dynamic changes. Directly from Kirchoff matrix, its eigenvalues can be used to ascertain how important each node is to maintain the overall mechanical connectedness of the network. This amounts to measuring how much the network Laplacian spectrum changes when the connections, or couplings, of a node with its neighbors are deleted. As such, the Mechanical Bridging Score (MBS) for a given mutation reflects the response ability of this residue. In the symmetrical stiffness matrix, the elements describe the effective spring constants associated with each residue pair. The stiffness for individual mutational residues is obtained by averaging all the elements in the corresponding row/column of the matrix. The detailed description of these dynamic features can be found in Bahar's work.[36] The statistical analysis of all features was performed in the R language (https://www.r-proj ect.org/, R Core Team, 2017), version 3.6.2. Plots were generated using R package ggplot2, version 3.3.3.[64] Statistical significance was determined by the Wilcoxon signed ranked test $(\mathrm{P}<0.01)$ in our analysis.

\section{Molecular Dynamics Simulations}

The MD simulations were performed on WT and mutant TNSALPs for the conformational dynamics study. To eliminate any unfavorable contacts, energy minimization was performed. Each structure was first minimized with the steepest descent algorithm and followed by the L-BFGS algorithm without any restraint. Total minimization was carried out until convergence, where the maximum atomic force was $<500 \mathrm{~kJ} / \mathrm{mol}-\mathrm{nm}$. The minimized 
structures were then slowly heated from 0 to $300 \mathrm{~K}$ over $100 \mathrm{ps}$ and equilibrated for an additional 200 ps. Later, MD simulations were further carried out for $100 \mathrm{~ns}$ using the GPU-enabled version of the GROMACS software package (version 5.1.4)[65] with the AMBER99SB-ILDN force field,[66] using a timestep of 2 fs. The structures were immersed in an octahedral box filled with TIP3P water molecules, imposing a minimum distance of 15 $\AA$ between the solute and the box. The non-bonded interaction potential was smoothly switched off between 10 and $12 \AA$, beyond which coulombic interactions were treated with the particle-mesh Ewald method.[67] The LINCS algorithm[68] was used to constrain the hydrogen containing bonds. A temperature of $300 \mathrm{~K}$ was maintained via the V-rescale dynamics.[69] The atomic positions were saved every 5000 steps (10 ps) for analyses. GROMACS analysis toolkit utilities were used to analyze MD trajectories produced during the last $70 \mathrm{~ns}$ production run to determine root mean square deviations (RMSDs), root mean square fluctuations (RMSFs) for each system.

\section{Allosteric Mutation Analysis}

Two methods were used for the investigation of allosteric effects of mutations. The dynamic network analysis was performed by the program, MD-TASK.[70] For each protein system, the network was constructed by extracting $\mathrm{C}_{\alpha}$ and $\mathrm{C}_{\beta}$ atoms of MD trajectories as nodes and the edge is created when two nodes are within $6.5 \AA$. Then, the allosteric communication pathways were calculated by connecting mutational sites and allosteric sites with the shortest edges, using the Floyd-Warshall algorithm.[71] AlloSigMA server[72, 73] was used to evaluate the allosteric effects of each mutation based on the Structure-Based Statistical 
Mechanical Model of Allostery. This statistical mechanical model estimates the allosteric free energy difference $\Delta \mathrm{g}$ of each residue used by the allosteric communication under mutation events to perform the allosteric mutation analysis in TNSALP.

\section{Results and Discussion}

\section{Sequence and Structural Landscape of ALPL mutations}

To clarify the sequential and structural characteristics of genotype and phenotype, we first divided all mutations into three categories according to the severity of HPP: the mild form, severe form, and control group. Here, 185 missense mutations related to 142 unique amino acids were found in the $A L P L$ gene, of which 111 mutations related to 100 residues were found to cause the mild form of HPP, while 74 mutations related to 63 residues were found to cause the severe form of HPP (Fig 2A). Moreover, 57 mutations related to 55 residue sites were found in asymptomatic people and, therefore, served as the control group. It should be noted that a residue may be related to different types of mutations (S1 Fig), while the Venn Diagram showed overlap of mutated residues of all three groups (Fig 2A). The primary sequence analysis showed that there are 261 conserved residues (conservation score >5, see S2 Fig) among the full length of 524 residues of the TNSALP monomer. In order to characterize the relationship between conservation and phenotype, we calculated the proportion of conserved residues in each group, and results are shown in Fig 2B. Among the mutation sites that cause severe phenotypes, more than $71.0 \%$ of the sites are highly conserved, while in the mild group, this proportion is $65.0 \%$, and in the control group, it is much lower, at only $27.3 \%$. It is worth noting that half of these highly conserved sites in the control group are from the 
intersection of the mild group and the severe group. Thus, we postulated a possible trend that mutations occurring at highly conserved sites are more likely to cause HPP disease.

A
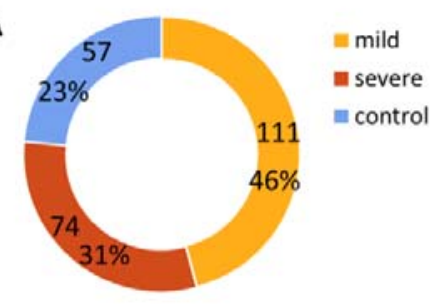

II mild

severe

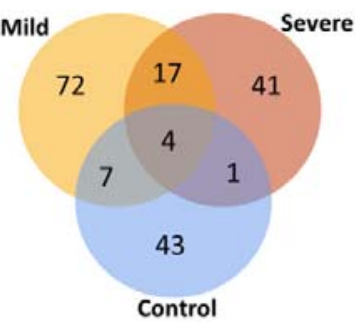

B

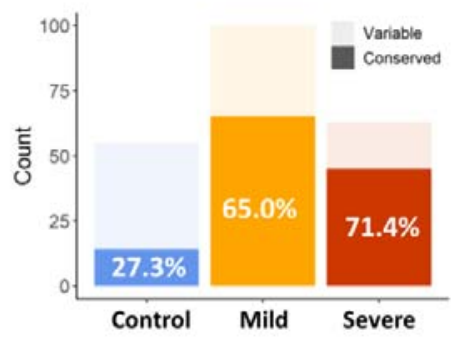

C
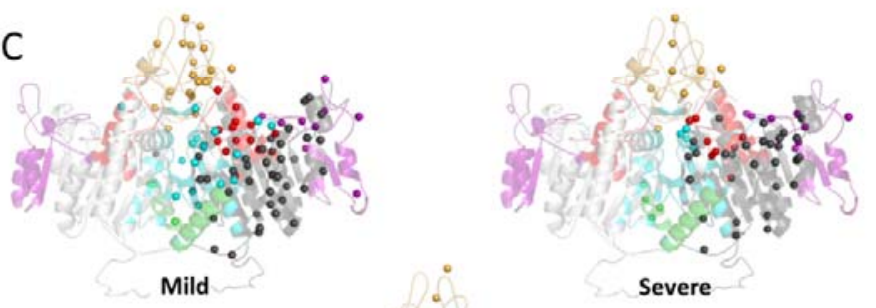

D

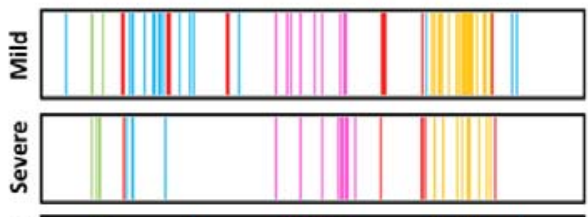

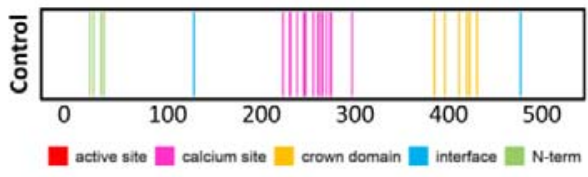

Control

Fig 2. Sequence and structural analysis of $A L P L$ mutations in the TNSALP protein. (A)

The composition and statistics of $A L P L$ mutations of based in the three categories: mild HPP, severe HPP, and control group. (B) The conservation distribution of mutations related to different phenotypes. (C) The structure of the TNSALP protein, and the distribution of mutation sites in the active site (red), calcium site (purple), crown domain (brown), dimeric interface (blue), and N-terminal domain (green). Missense mutations in the three groups are shown as colored spheres based on the coloring scheme of the domains to which they belong. (D) Distribution of different clinical phenotypic mutations across the length of the TNSALP protein. 
The 3D structure of TNSALP was then computationally modeled by using the crystal structure of human placental alkaline phosphatase as the template (S3 Fig). By mapping all the mutations onto the modeling TNSALP structure, we identified the spatial distribution of the three kinds of mutations (Fig 2C and 2D). It was found that 104 out of 185 (66\%) pathogenic mutations were located at these five functional domains; in particular, $17 \%$ were at the crown domain, while only 28 of 57 (49\%) mutations in the control group were distributed in this domain. In the mild group, 12 mutations were located at the active center, 21 at the interface, 23 at the crown domain, 9 at the $\mathrm{Ca}^{2+}$ binding site, and 3 at the N-terminal. The distribution of mutations in the severe group is as follows: 7 mutations at the active center, 4 at the interface, 9 at the crown domain, 12 at the $\mathrm{Ca}^{2+}$ binding site, and 4 in N-terminal (Fig 2D). On the other hand, in the control group, 16 mutations were located at the $\mathrm{Ca}^{2+}$ binding site, 2 at the interface, 6 at the crown domain and 4 at the N-terminal. The major difference in the spatial distribution between pathogenic and control groups was that the control group had no mutations at the active site, thereby suggesting the role of active sites in HPP pathogenicity.

\section{Structural Environment and Evolutionary Properties of ALPL Mutations}

The overall sequence and structural landscape of $A L P L$ mutations suggest that evolutionary conservation and spatial distribution of mutation sites in the TNSALP protein may be linked to different functional roles in disease phenotypes. Here, we performed the comparative 
RASA and evolutionary calculation of TNSALP for the large-scale analysis of $A L P L$ mutations. These physicochemical features could impart a particular structural environment and evolutionary properties to mutational hotspot sites. According to the RASA (Fig 3A), residues were classified into three classes, i.e., buried areas (RASA $<5 \%$ ), semi-exposed areas (between 5\% and 30\%), and fully exposed areas (RASA>30\%). Although different types of mutations were distributed across all functional domains, disease-causing variants are more likely to occur at the buried areas (122 mutations, 65.9\%). By contrast, nonsense variants are more likely to occur at the surface (32 mutations, 56.4\%). We systematically characterized the evolutionary landscape of $A L P L$ mutations; the distribution of the three types of mutations on $\boldsymbol{S}(i)$ and $\boldsymbol{M I}$ profiles are shown in Fig 3B and C, respectively. Regarding conservation, 178 of the 524 amino acid residues in the protein sequence are relatively conserved $(\boldsymbol{S}(i)<1.0)$. In general, disease-causing variants are more likely to occur at the conserved sites (99 mutations out of 185 pathogenic mutations with $S(i)<1.0,53.5 \%)$, while the nonsense variants are more likely to occur at the less conservative sites (44 mutations out of 57 neural mutations with $\boldsymbol{S}(i)>1.0,77.2 \%)$. We observed a significant positive correlation between co-evolution and entropy (S4A Fig), and the distributions of $\boldsymbol{S}(i)$ and $\boldsymbol{M I}$ scores for mutations were also very similar. By using $\boldsymbol{M I}=0.2$ as a cutoff, the low values of amino acids correspond to residues that were highly co-evolutionary. For pathogenic mutations, 64.9\% (48 out of 74) severe mutations and $55.9 \%$ (62 out of 111) mild mutations occurred at highly co-evolutionary residues, whereas for the control mutations, only $28 \%$ belonged to co-evolutionary residues. 

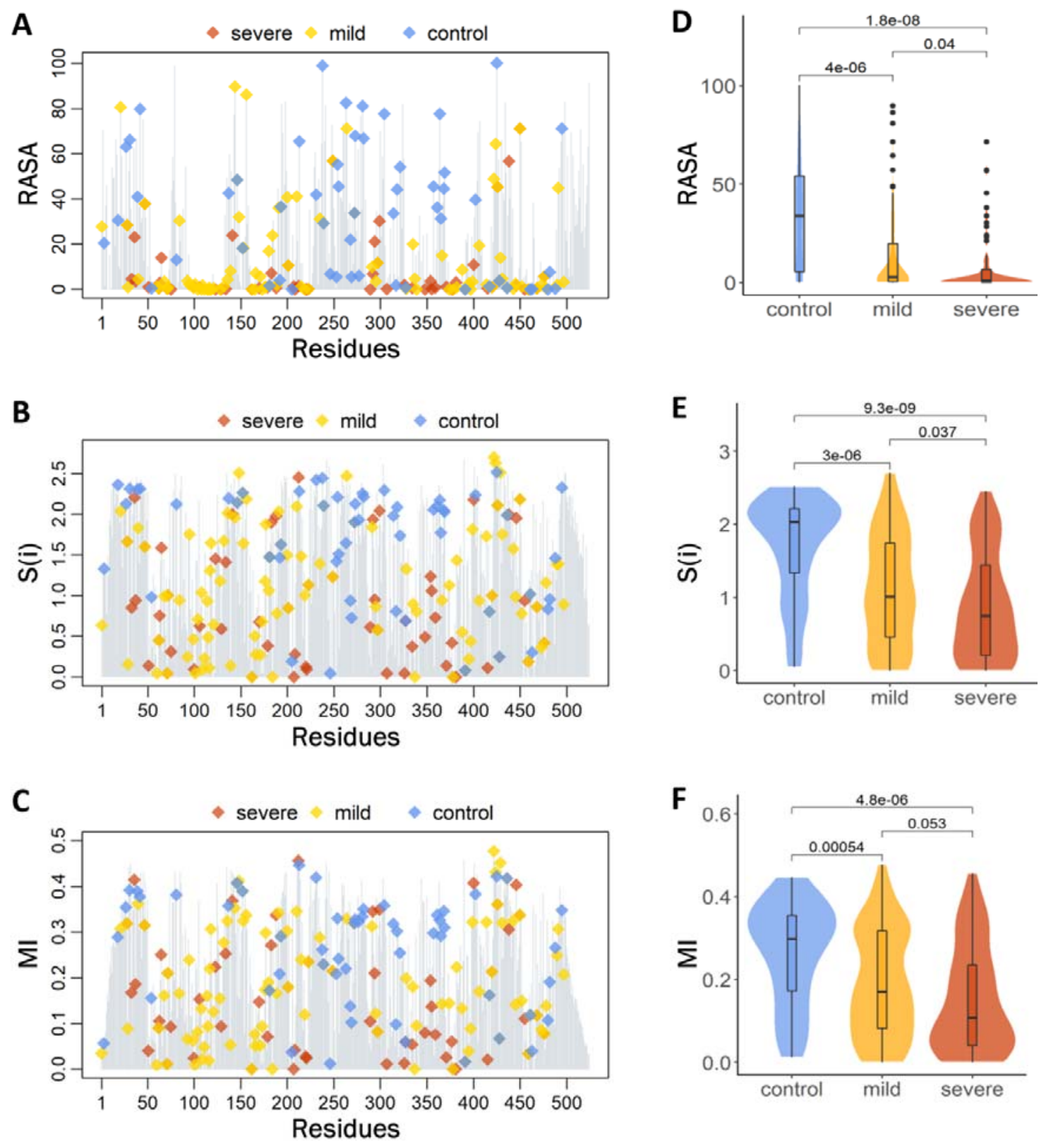

Fig 3. The (A) relative solvent accessible area (RASA), (B) entropy ( ) and (C) co-evolutionary $(M I)$ profiles for each residue in TNSALP; mild, severe, and control mutations are highlighted as yellow, red, and blue diamonds, respectively. The comparison of (D) the RASA, (E) and (F) MI, between the control group mutations and mild and severe mutations. Statistical significance was determined by the Wilcoxon signed-ranked test, with $P$ values $<0.01$. 
The statistical analysis of RASA, $\boldsymbol{S}(i)$ and $\boldsymbol{M I}$ for control, mild, and severe mutations showed statistical significance between the control and mild, and control and severe mutations; however, there was no statistical significance between the mild and severe mutations (Fig 3D-F). Accordingly, structural environment and evolutionary studies have found that the HPP-causing ALPL mutations, especially severe mutations, are located in buried, conserved and co-evolutionary residues with small RASA, $\boldsymbol{S}(i)$, and $\boldsymbol{M I}$ scores.

\section{Energetic Analysis Reveals the Effect of Severe and Mild Mutations on TNSALP Stability}

To explore the thermodynamic determinants of mutational hotspots and establish a robust baseline for ranking and evaluating functional significance of mutational hotspots, FoldX[56] was used for the prediction of free energy changes in single point mutations. In this method, a mutation is predicted when there is a destabilization effect positive value of Gibbs free energy changes $(\Delta \Delta \mathrm{G})$, while the respective mutation is a stabilized protein with a negative value of $\Delta \Delta \mathrm{G}$. As shown in Fig 4(A), we found that most of the disease mutations are associated with decrease in binding affinity $(\Delta \Delta \mathrm{G}>0)$, reducing the protein stability. Several mutations showed significant peaks corresponding to the severe phenotype, which were located in different domains, such as G220R, G326R, G326V, G221R, G221V, D378H, G129, G334D, C201Y, R223W, and G63R. We also noticed that the largest destabilization effect upon substitution was found for mutations in the mild group, namely G339R, P108L, A468V, G491R, C139Y, A111T, R428Q, A114T, and G249V. On the other hand, the mean value of $\Delta \Delta \mathrm{G}$ for most 
mutations in the control group, such as L365M, K27S, V314E, S368Q, R272C, R246S, L18F, and $\mathrm{Y} 39 \mathrm{~N}$ was $<1.0 \mathrm{kcal} / \mathrm{mol}$. Furthermore, as shown in Fig 4B, we found that the predicted folding free energy change $\Delta \Delta \mathrm{G}$ of mutations which cause severe phenotype (mean $\Delta \Delta \mathrm{G}$ was $5.82 \mathrm{kcal} / \mathrm{mol}$ ) was obviously higher than that in the mild group (mean $\Delta \Delta \mathrm{G}$ was 2.05 $\mathrm{kcal} / \mathrm{mol}, P=2.4 \mathrm{e}-06$ ), and much higher than that in the control group (mean $\Delta \Delta \mathrm{G}$ was 0.43 $\mathrm{kcal} / \mathrm{mol}, P=1.5 \mathrm{e}-11)$. Therefore, most of the disease-causing mutations are associated with decrease in binding affinity, and this trend varies in different phenotypes.

In addition, $\Delta \Delta \mathrm{G}$ provides a benchmark or metric that can be used to compare with physicochemical properties for large-scale analysis of other mutations. We have compared the $\Delta \Delta \mathrm{G}$ with the RASA, $\boldsymbol{S}(i)$ and $\boldsymbol{M I}$, and weak correlations were found among them (S4B-D Fig), with negative coefficients of $0.285,0.350$ and 0.321 , respectively. These low coefficients imply that the three properties provide additional measures of $\triangle \triangle \mathrm{G}$ for describing $A L P L$ mutations, namely pathogenic mutations tend to have a high $\Delta \Delta \mathrm{G}$ value and low RASA, $\boldsymbol{S}(i)$, and MI values. For example, pathogenic mutations, including G220R, G221R, G326R, G129R, P108L, R223W, and G63R (severe mutations), and G339R and A468V (mild mutations) showed obvious energy change but with low RASA, $\boldsymbol{S}(i)$, and $\boldsymbol{M I}$ scores. By contrast, several nonsense mutations, such as L18F, R272C, H281S, E146K, and T273M, were predicted to have small $\Delta \Delta \mathrm{G}$ but with high RASA, $\boldsymbol{S}(i)$, and $\boldsymbol{M I}$ scores. Based on the above analysis, we concluded that most of the "severe" and "mild" mutations were predicted to have large $\Delta \Delta \mathrm{G}$ values and differed in terms of evolutionary conservation and physicochemical properties. 


\section{A}

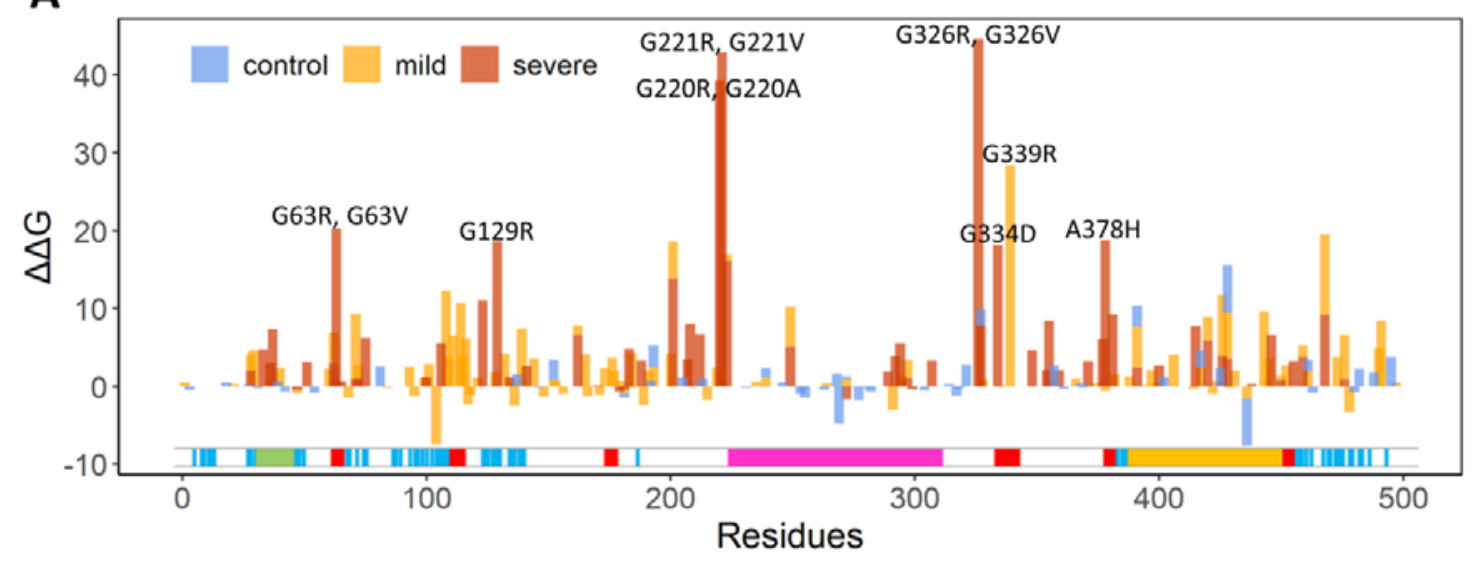

B
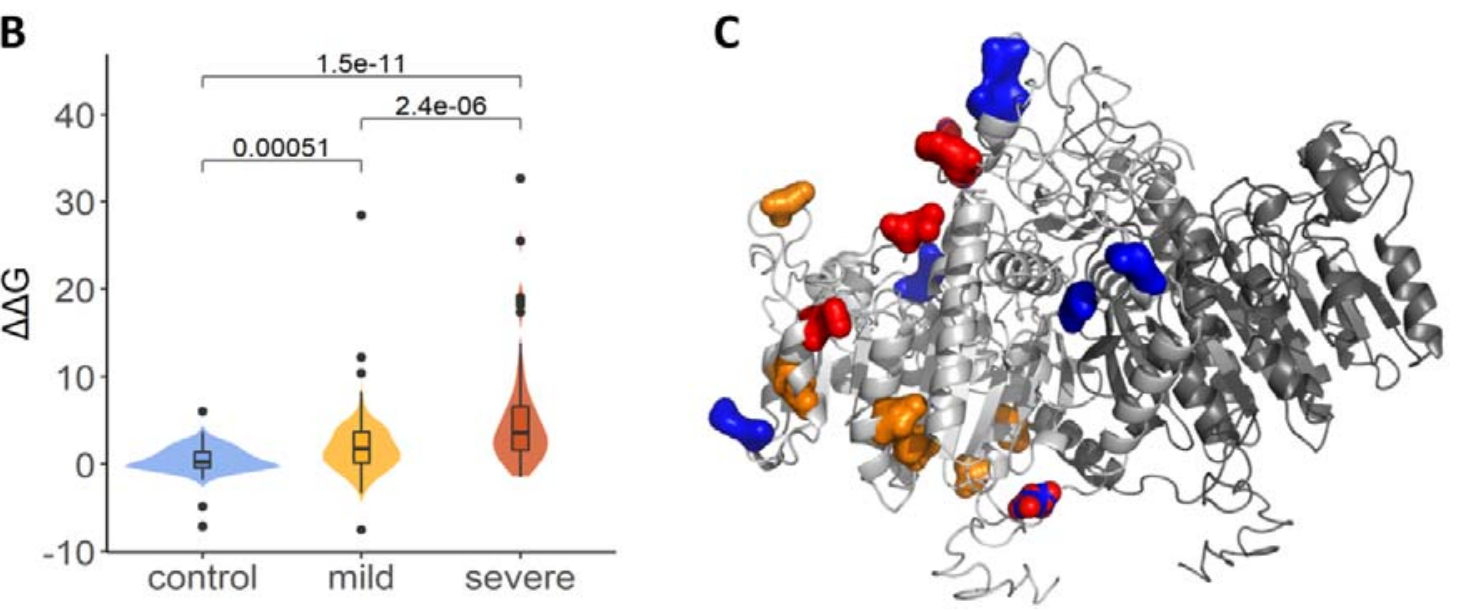

Fig 4. (A) The free energy changes induced by single point mutations. The profiles of mild, severe, and control mutations are shown as yellow, red and blue bars, respectively. (B) The statistical difference between predicted folding free energy change of $A L P L$ single residue variations in the control, mild, severe groups was measured by Wilcoxon. test, with $P$ values< 0.01. (e) The structural distribution of disease-causing mutations with low $\Delta \Delta \mathrm{G}$.

However, some mutations were not consistent with the above results. In the severe group, mutations including R272L, N47I, L299P, R272H, H438L, and R450C, the $\Delta \Delta \mathrm{G}$ values of many mutations were $<1.0 \mathrm{kcal} / \mathrm{mol}$, while the RASA score was $>30 \%, \quad>1.0$, and $\mathrm{MI}>$ 0.2. Mild group mutations, such as R272H, R450H, V424A, K264R, E21K, V424M, R184Q, 
E84V, N47I, K422R, and T148I showed a slight energy change $(\Delta \Delta \mathrm{G}<1.0 \mathrm{kcal} / \mathrm{mol})$ but displayed high co-evolutionary conservation $(\boldsymbol{S}(i)>1.0, \mathrm{MI}>0.2)$ and RASA score $(>30 \%)$. By contrast, some nonsense mutations such as I269V, H482N, F327C, A488S, T277A, T255I, I317L, and D239Y, were predicted to have a large energy change $(\Delta \Delta \mathrm{G}>1.0 \mathrm{kcal} / \mathrm{mol})$ with low co-evolutionary conservation scores $(\boldsymbol{S}(i)<1.0$, MI $<0.2)$ and RASA score $(<30 \%)$, Structurally these mutations are distributed at different domains and not clustered together (Fig 4C). These results highlighted potential limitations of using only the folding free energy $(\Delta \Delta \mathrm{G})$ changes to predict pathogenic mutations, thus suggesting that molecular disease mechanisms may be determined not only by local energetic changes but through impairment of long-range allosteric interactions and alterations in the global interaction networks. In addition, the random structural distribution of disease-causing mutations calls for a structural systems biology approach for predicting the effect of different types of mutations, toward the understanding of their genotype-phenotype relationships.

\section{Network Modeling of $A L P L$ Mutations}

Network theory has become a widely used method to quantify protein structures and functions in terms of their topological connectivity.[74] In our analysis, we used the difference between network centrality measures of mutant and WT values to describe the topological change of TNSALP caused by ALPL mutations. For each amino acid substitution, four common network parameters including degree $\left(\mathrm{K}_{\mathrm{i}}\right)$, betweenness $\left(\mathrm{B}_{\mathrm{i}}\right)$, closeness $\left(\mathrm{C}_{\mathrm{i}}\right)$, and clustering coefficient $\left(\mathrm{C}_{\mathrm{Ci}}\right)$ were calculated. The computational results of $\Delta \mathrm{K}, \Delta \mathrm{B}, \Delta \mathrm{C}$, and $\Delta \mathrm{C}_{\mathrm{C}}$ associated with 
each mutation in the mild, severe, and control groups, are illustrated in Fig 5 and 6.

A

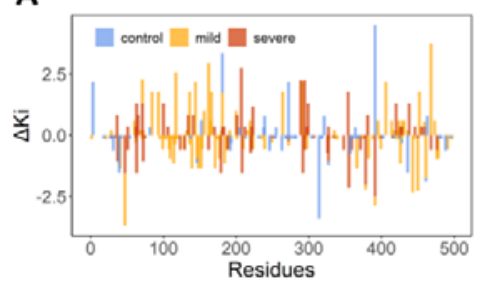

D

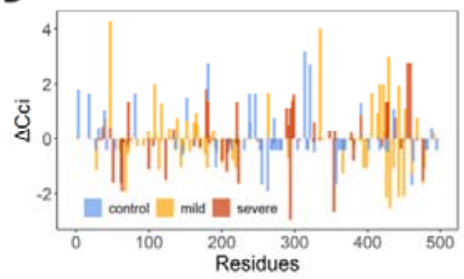

G

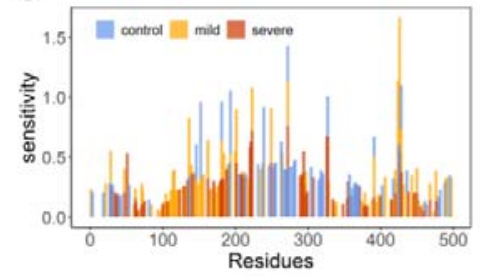

B
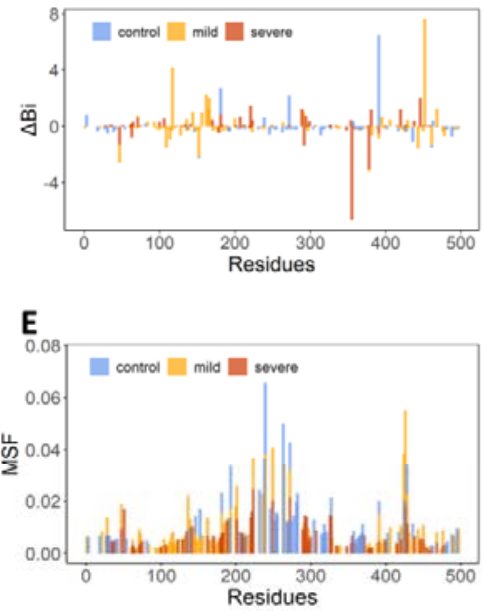

H

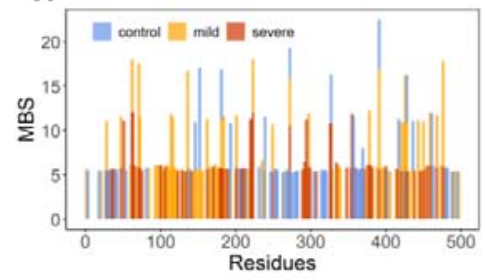

C

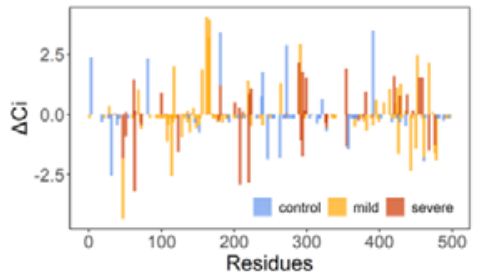

F

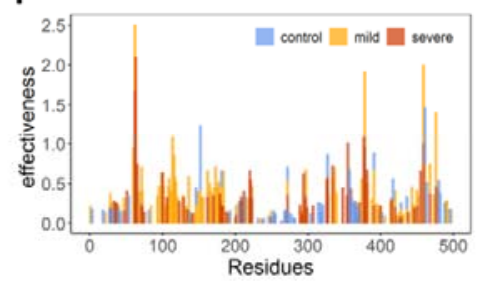

I

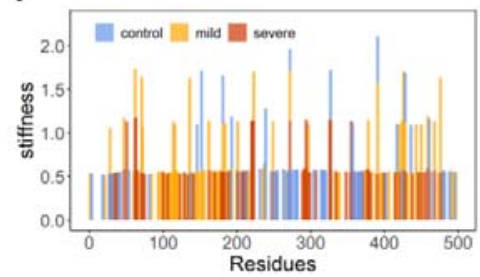

Fig 5. Network topological and dynamic analysis of mutations in $A L P L$. (A) $\Delta \mathrm{K}$, (B) $\Delta \mathrm{B}$,

(C) $\Delta \mathrm{C}$, (D) $\Delta \mathrm{Cc}$, (E) MSF, (F) Effectiveness, (G) Sensitivity, (H) MBS, and (I) stiffness profiles for the three types of mutations. Control, mild and severe mutations are represented as blue, orange, and red bars, respectively.

The profiles in Fig 5 (A-D) depict that most of the mutations show a wide range of changes in the values of $\mathrm{K}_{\mathrm{i}}, \mathrm{C}_{\mathrm{i}}$, and $\mathrm{C}_{\mathrm{Ci}}$. In general, some common peaks were found in $\Delta \mathrm{K}$, $\Delta \mathrm{B}$, and $\Delta \mathrm{C}$ profiles, such as the severe mutation $\mathrm{R} 391 \mathrm{H}$ and three mild mutations $\mathrm{G} 162 \mathrm{~S}$, T166I, and R272C. Detailed analysis of the $\Delta \mathrm{K}$ profile showed that its peaks corresponded to pathogenic mutations, including severe mutations R391C, Q207P, D294A, L289F and mild mutations A468V, G162S, Y117C, G144E, E291K, and D406G. These significant peaks 
aligned precisely with functional centers, including hotspot positions involved in the crown domain, the homodimer interface, and the $\mathrm{Ca}^{2+}$ binding site, thereby suggesting their latent functional roles. In comparison, smaller number of variations were observed in the $\Delta \mathrm{B}$ profile (Fig 5B), but much sharper peaks for severe mutations F355I, D378H and A446T and mild mutations E452K, Y117C, G162S, R152C, and T166I were found. Functional domain analysis revealed that E452 and D378, R391, and A446, R272 are involved in the active site, the crown domain, and the $\mathrm{Ca}^{2+}$ binding site, respectively. Eleven peaks were found in both the $\Delta \mathrm{C}$ and $\Delta \mathrm{Cc}$ profiles. In the $\Delta \mathrm{C}$ profile (Fig 5C), eight corresponded to pathogenic mutations, including G162S, T166I, G63R, T165I, L208F, E291K, G220R, and N47I, and only one (E291) was located at the functional domain. In the $\Delta \mathrm{Cc}$ profile (Fig 5D), the other set of pathogenic mutations, peaks corresponded to R335T, N47I, E429K, D294Y, G455D, V459F, E354D and V431A, including many functional sites. Among these, R335 and G455 were located at the active site, D294 and Y263 at the $\mathrm{Ca}^{2+}$ binding site, and E429 and V432 at the crown domain.

On the statistical comparison of the topological differences between the three groups of mutations, it was observed that four network parameters were decreased for most groups of mutations (with mean values <0), only $\boldsymbol{B}_{i}$ and $\boldsymbol{C}_{i}$ were increased for the severe mutations. In addition, both $\Delta \mathrm{B}$ and $\Delta \mathrm{C}$ showed significant differences between any two groups of mutations (Fig 6). As shown in Fig 6 (B), the mean values of $\Delta \mathrm{B}$ for the severe, mild, and control groups are $0.05,0.16$ and 0.21 , respectively, with $P=1.889 \mathrm{e}-07,9.906 \mathrm{e}-09$, and 0.0005129 , respectively, by the Wilcoxon signed rank test between the severe and mild, severe and control, and mild and control groups. 


\section{A}
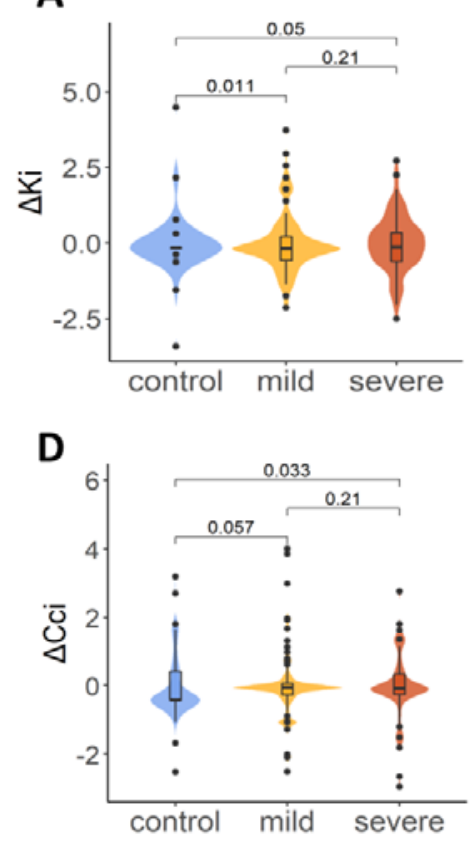

G

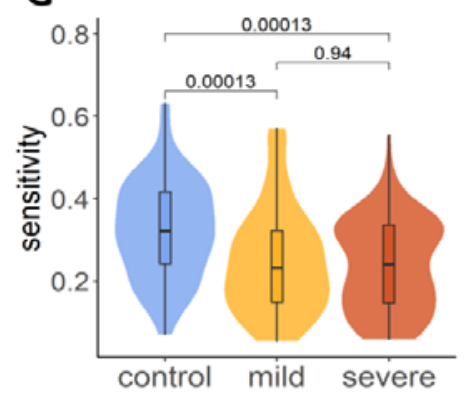

B

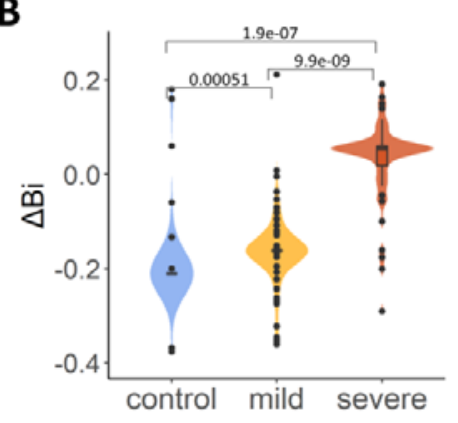

E

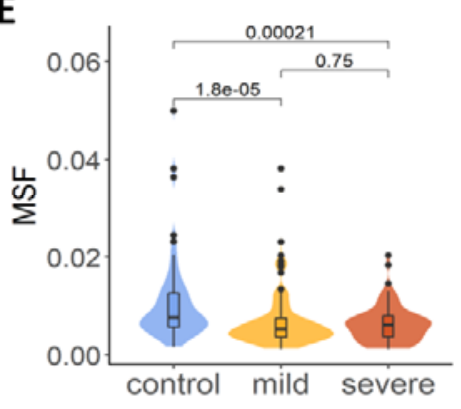

H

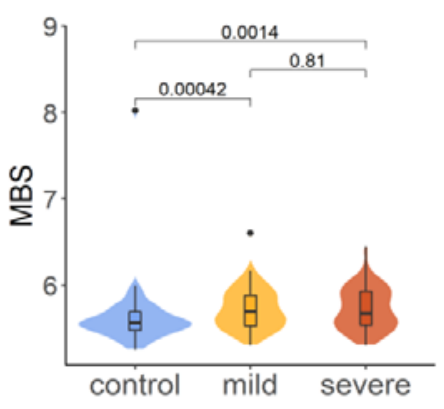

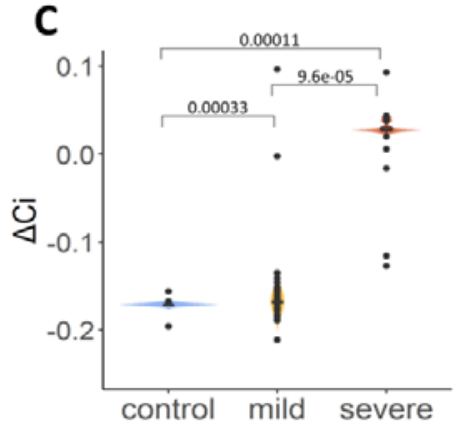
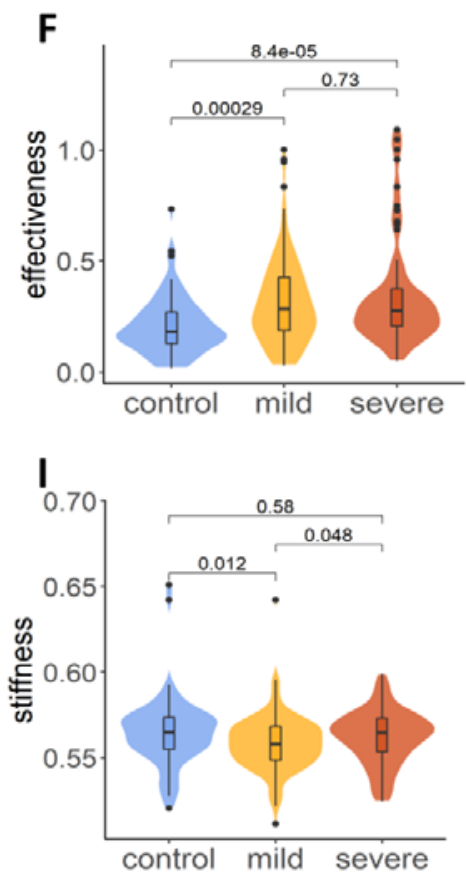

Fig 6. Comparison of the change of four network centralities and five dynamics-based parameters, including (A) $\Delta \mathrm{K}$, (B) $\Delta \mathrm{B}$, (C) $\Delta \mathrm{C}$, (D) $\Delta \mathrm{Cc}$, (E) MSF, (F) Effectiveness, (G) Sensitivity, (H) MBS, and (I) stiffness, among mutations in the control, mild, and severe groups by Wilcoxon signed ranked test.

In contrast, as shown in Fig $6(\mathrm{C})$, the mean values of $\Delta \mathrm{C}$ for the severe, mild, and control groups are $0.03,0.17$ and 0.17 , with $P=9.646 \mathrm{e}-05,0.0001094$, and 0.0003274 , respectively, by the Wilcoxon signed rank test, between the severe and mild, severe and control, and mild 
and control groups. Through this network centrality analysis, we found that pathogenic $A L P L$ mutations showed significantly higher variation in $\Delta \mathrm{B}$ and $\Delta \mathrm{C}$ compared with the control group. As two network global topological measures, the significant change in betweenness and closeness suggests that pathogenic mutations (especially severe mutations) may affect the overall connectively of the interacting network rather than the local geometry. In particular, $\Delta \mathrm{B}$ showed the best discrimination, as has been suggested, and could serve as a potential in-silico marker for pathogenic NaV1.7 mutations.[75] Remarkably, the increase of betweenness and closeness caused by severe mutations may suggest a structural and functional change of the protein, by tightening the correlated dynamical network and making the severe state as a more allosteric molecular machine.

\section{Elastic Network Modeling Highlights Mutational Signatures of Conformational Flexibility}

In addition to sequence conservation and structural information, structural dynamics has also been shown to be another determinant of the functional impact of missense variants. A series of ENM-based dynamics descriptors, including GNM-based MSF, PRS-based effectiveness and sensitivities, and ANM-based mechanical bridging score (MBS) and stiffness, have been introduced to enhance the predictive ability of disease-related mutations[36]. Here, we employed these dynamic features to systematically characterize different types of $A L P L$ mutational sites.

First, mapping the three types of mutations onto the dynamic profiles based on the TNSALP protein, provided primary insight into the interpretation of the functional impact of 
variants in the light of the intrinsic dynamics of the mutational site (Fig 5 E-I). As shown in the MSF profile (Fig 5E), pathogenic mutations including both mild and severe mutational sites always demonstrate lower MSF values, except some pathogenic mutations at the $\mathrm{Ca}^{2+}$ binding domain. Some particular pathogenic mutations, located at minimal positions that correspond to hinge sites, have been found, such as M62, G63, S65, A377, D378, and H381 belong to severe mutational sites, and D60, V461, and G473 correspond to mild mutational sites. Structurally, these hinge mutational hotspots are located at the active site and the dimer interface. These conformational dynamic signature of disease-associated mutations have been revealed both at the genome[76] and proteome-levels.[31]

Regarding the PRS analysis, two matrices were used to quantify the allosteric effect of each residue, which is effectiveness and sensitivity, for evaluating the propensity of residues to act as sensors or as effectors of allosteric signals. As shown in profiles in Fig 5F and G, the distribution of effectiveness and sensitivity for $A L P L$ mutation hot spots shows different trends. In general, most of the pathogenic mutations match almost exactly with some peaks of effectiveness profile, with sharp dominant peaks being found for three severe mutational sites (G63, A377, and V459). On the other hand, the peaks of the sensitivity profile correspond to some control mutational sites, while pathogenic mutational sites have smaller sensitivity, such as V95 and G473 in the mild group and G63, S65, and V459 in the severe group. As two unusual ENM-based dynamics features, the distribution of mutations in MBS and stiffness profiles are shown in Fig $5 \mathrm{H}$ and I. For the MBS profile, the overall distribution of pathogenic mutational sites is larger than that of control mutational sites, highlighting the importance of pathogenic mutational sites in maintaining the stability of the $A L P L$ protein. 
Lastly, the mapping of mutational sites onto stiffness demonstrates the relatively uniform distribution; thus, it is not easy to find hits to distinguish severe, mild, and control mutational sites by only considering mechanical stiffness.

In order to clearly observe the ability of dynamic parameters to distinguish mutations in different groups, statistical analysis was further performed on the data of severe, mild, and control mutational sites. As shown in Fig 6 (E), the control group had the highest mean MSF, with $P=1.8 \mathrm{e}-05$ and 0.00021 by the Wilcoxon signed rank test, in comparison with mild and severe mutations, showing the significant differences between both pathogenic mutational sites and the control group. However, significant differences were not found between mild and severe mutational sites, with $P=0.75$. Similar significant results were also found for effectiveness (Fig 6F), sensitivity (Fig 6G), and MBS (Fig 6H), while significant differences in stiffness could not be found among the three groups of mutations $(P>0.01$, Fig 6I).

In the present section, we have compared the conformational dynamics of the three types of $A L P L$ mutations in terms of five ENM-based features. In general, the disease-related mutational sites are shared by lower conformational flexibility and propensity to act as sensors, but have high propensity to act as effectors and key nodes in maintaining stability. This suggests that the inclusion of ENM-based dynamics descriptors may increase the prediction of pathogenic mutations in $A L P L$.

\section{Machine Learning Analysis Determines Key Molecular Features for Classifying Different Mutation Types}


In order to find the relationship between the above studied features, their redundancy and ability to classify and predict various mutation types, we first computed the pairwise correlations between different prediction scores by using Spearman's rank correlation coefficient (Fig 7A). It is easy to comprehend that these features can be classified into three major types according to their related sequence, network, and dynamics information. Among sequence-based features, $\boldsymbol{S}(i)$ has high positive correlations with $M I$ and RASA, but these three features all have negative correlation with conservation calculated by Consurf. For the four network parameters, $\Delta \mathrm{K}, \Delta \mathrm{B}$ and $\Delta \mathrm{C}$ show significant correlations between each other; only $\Delta \mathrm{Cc}$ is an independent factor. The dynamics-based matrices that show more complex correlations, have been divided into two groups: one includes stiffness, MSF, and sensitivity, and the other includes effectiveness and MBS. A highly positive correlation was predicted within each group, but a negative correlation was found between the two groups. The special index $\Delta \Delta \mathrm{G}$ did not show any correlation with other features. Accordingly, this suggests that these features could complement our understanding of the molecular signatures of HPP-causing mutations. 


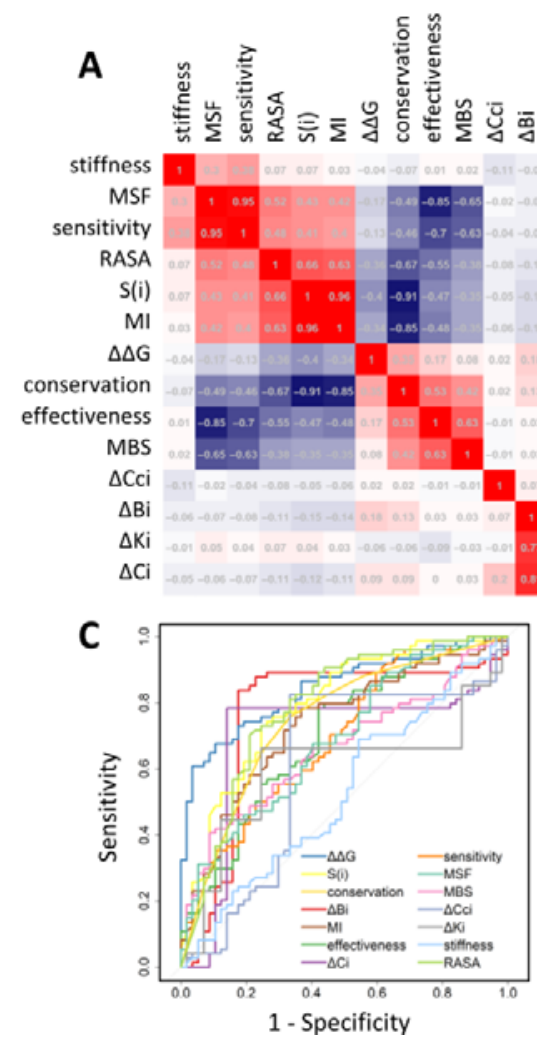

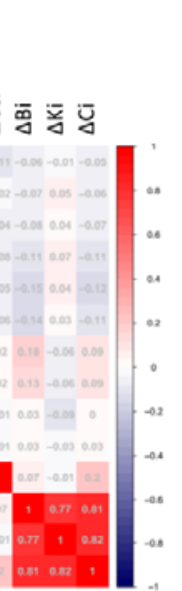

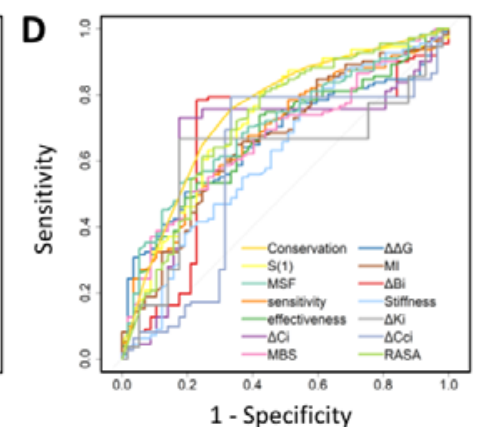

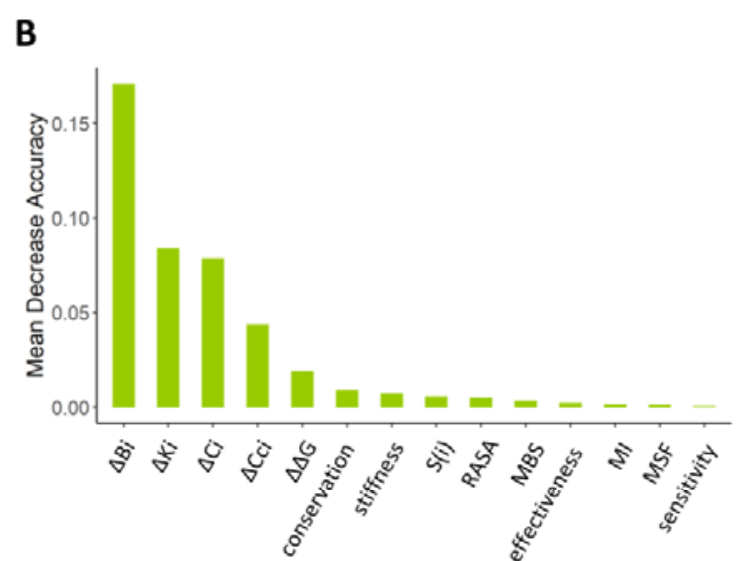

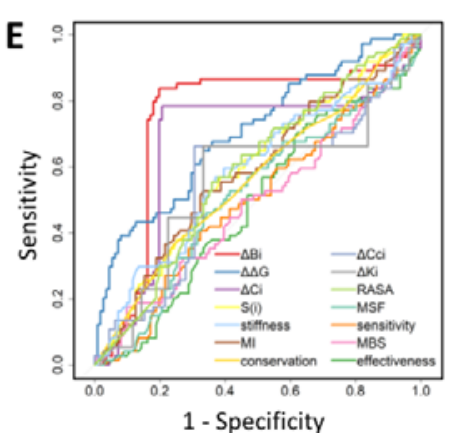

Fig 7. Performance evaluation of feature classification. (A) The heat map of pairwise

Spearman's rank correlation coefficients between different features. (B) Feature importance of all used features ranked based on Mean Decrease Accuracy in the RF classification. ROC curve AUCs for 14 features as a function of 1-specificity, including ROC curves for evaluating each feature in classifying $A L P L$ mutations between (C) the control and severe groups, (D) control and mild groups, and (E) mild and severe groups.

Next, we employed a RF model to classify mild, severe, and control groups using the 14 features mentioned above (See S1 Text for detail method). Through the RFE algorithm, we considered classifying the model containing five variables, whose accuracy of repeated cross-validation was $80.5 \%$, as the optimized one, which was used for comparison with the model using all features. To be more specific, the feature importance was ranked by the mean 
decrease accuracy (Fig 7B). Most interestingly, we found that the top four important features were all network-based features, including $\Delta \mathrm{K}, \Delta \mathrm{B}, \Delta \mathrm{C}$, and $\Delta \mathrm{Cc}$, suggesting an essential role of protein network topology in controlling mutation events. $\Delta \Delta \mathrm{G}$ is an important feature and is also an important determinant of protein stability in mutations. To our surprise, features relating to ENM protein dynamics were not ranked as important predictors. This may be caused by the loopy structure of the TNSALP protein. By considering only the top five features, the RF model yielded an accuracy of 96.8\%. However, taking all features into account, the accuracy of the RF model dropped to $92.2 \%$. The inclusion of more features reduces the predictive accuracy of the RF model, which led us to focus on the specific interpretability of each parameter.

Table 1. The AUC of 14 characteristics or parameters in each comparison group. The characteristics that performed best in each group are highlighted in red.

\begin{tabular}{lccc}
\hline Features & Mild vs control & Severe vs control & Mild vs severe \\
\hline conservation & $\mathbf{0 . 7 4 9 3}$ & 0.7927 & 0.5632 \\
$\mathrm{~S}(\mathrm{i})$ & 0.7204 & 0.7934 & 0.5906 \\
$\mathrm{MI}$ & 0.6633 & 0.7336 & 0.5841 \\
$\mathrm{RASA}$ & 0.5284 & 0.5043 & 0.5329 \\
$\Delta \Delta \mathrm{G}$ & 0.6641 & $\mathbf{0 . 8 4 4 7}$ & 0.7048 \\
$\Delta \mathrm{K}_{\mathrm{i}}$ & 0.6185 & 0.5991 & 0.5538 \\
$\Delta \mathrm{B}_{\mathrm{i}}$ & 0.6618 & 0.7636 & $\mathbf{0 . 7 4 7 1}$ \\
$\Delta \mathrm{C}_{\mathrm{i}}$ & 0.6675 & 0.6961 & 0.6682 \\
$\Delta \mathrm{Cc}_{\mathrm{i}}$ & 0.5886 & 0.6081 & 0.5539 \\
$\mathrm{MSF}$ & 0.7023 & 0.6893 & 0.5140 \\
effectiveness & 0.6709 & 0.7009 & 0.4850 \\
sensitivity & 0.6802 & 0.6957 & 0.4968 \\
MBS & 0.6666 & 0.6632 & 0.4893 \\
stiffness & 0.619 & 0.5281 & 0.5860 \\
\hline
\end{tabular}

Toward the interpretability of the classification effect of each parameter, we further 
evaluated the performance of the 14 features of the dataset composed of mild and severe mutations, mild and control mutations, severe and control mutations. To this end, the ROC curves with the AUCs of the 14 metrics for each comparison group were plotted, and the related values are listed in Table 1. First, we examined the difference in the AUCs for the 14 functional features of the severe and control groups (Fig 7C); $\Delta \Delta \mathrm{G}$ showed appreciable performance with AUC 0.8447. Moreover, the $\boldsymbol{S}(i)$, conservation score, $\Delta \mathrm{B}$ also showed good performance with AUCs, all greater than 0.73. We then tested the performance of the 14 features between mild and control group mutations (Fig 7D), as well as the mild and severe group mutations (Fig 7E). In the classification of the mild group and control group, two sequence-based and four dynamics-based parameters showed moderate predictive performance. The AUC of the conservation score, entropy, MSF, effectiveness, sensitivity, and MBS were $0.7493,0.7204,0.7023,0.6709,0.6802$, and 0.6666, respectively. By contrast, in the classification of the mild and severe groups, we found that $\Delta \mathrm{B}$ showed the best performance, with an AUC $=0.7471$, while, $\Delta \Delta \mathrm{G}$ showed the second-best performance, with an $\mathrm{AUC}=0.7048$

In the features analysis, we argue that protein sequence, structure and dynamics all play important roles in the understanding of $A L P L$ mutations and their resultant phenotypes. In our study of ALPL mutations, the RF model only included network topological and $\Delta \Delta \mathrm{G}$ features that can predict the phenotypic severity of hypophosphatase. The most interesting finding is that $\Delta \Delta \mathrm{G}$ is a good indicator for identifying disease mutations, while $\Delta \mathrm{B}$ is the best indicator for classifying mild and severe mutations. 


\section{Atomistic Simulations and Network Modeling Determine the Effects of Severe Phenotype-Associated Mutations on Allosteric Communications}

Based on previous results, some severe phenotype-related mutations have relatively low $\Delta \Delta \mathrm{G}$ but higher $\Delta \mathrm{B}$ values in network topology. To gain energetic and topological insights, we have compared $\Delta \mathrm{B}$ and $\Delta \Delta \mathrm{G}$ values for all mutations. The scatterplot revealed that N47I, L289F and M355I were three severe mutations with large $\Delta \mathrm{B}$ but low $\Delta \Delta \mathrm{G}$ (Fig 8A). In order to determine the dynamics effects induced by severe phenotype-associated mutations, we performed a set of four independent, all-atom MD simulations for WT and three mutant TNSALPs caused by N47I, L289F, and M355I variants (Fig 8). The RMSD values of C $\alpha$ atoms served as an overall measurement of the departure of the structures from the initial coordinates (S5A Fig). All the systems became for a duration of $30-50 \mathrm{~ns}$, and the RMSD values became stable during the last $50 \mathrm{~ns}$ of the simulations. The conformational dynamics results (S5B Fig) revealed that the profile of RMSF is very consistent in the WT and mutant systems, and no conformational change occurred at the active site, further explaining why $\Delta \Delta \mathrm{G}$ for these severe mutations were small. The differential fluctuation $\Delta \mathrm{RMSF}$, with respect to WT, showed considerable changes in regions around the ion binding pocket and the $\mathrm{Ca}^{2+}$ binding site located in the other monomer, with two of the largest peaks at residues, N190 and S258 (Fig 8B). This suggests the existence of allosteric communication between the mutant sites and these two regions. Accordingly, in the four systems, we selected mutant sites in the chain A as starting points and N190 and S258 of the chain B as the target residues to further identify specific allosteric pathways caused by severe phenotype-associated mutations. 
The shortest path algorithm based on dynamic network models was employed to identify specific allosteric pathways. As shown in Fig 8 (C-F), the inter-molecular interaction of A: G473-B: V95 was predicted as the key bridge in WT, and the other interaction of A: G386-B: Q106 as the key bridge in mutants. It was, therefore, determined that WT and mutant TNSALPs use different allosteric interfaces for signal transmission. Overall, pathway plasticity was found to exist, although the structures between WT and mutants were well conserved, conforming to the recent function-centric allosteric regulation study.[77] Among these pathways, a common region including three active site residues $(\mathrm{T} 100 \rightarrow \mathrm{P} 108 \rightarrow \mathrm{D} 109)$ corresponding to mutational sites was also found, suggesting that these mutational paths may lead to the same functional state. In particular, the molecular signatures showed that T100, P108, and D109 have relatively high effectiveness and $\Delta \mathrm{B}$, demonstrating that these nodes are key sites for structural signal propagation. 

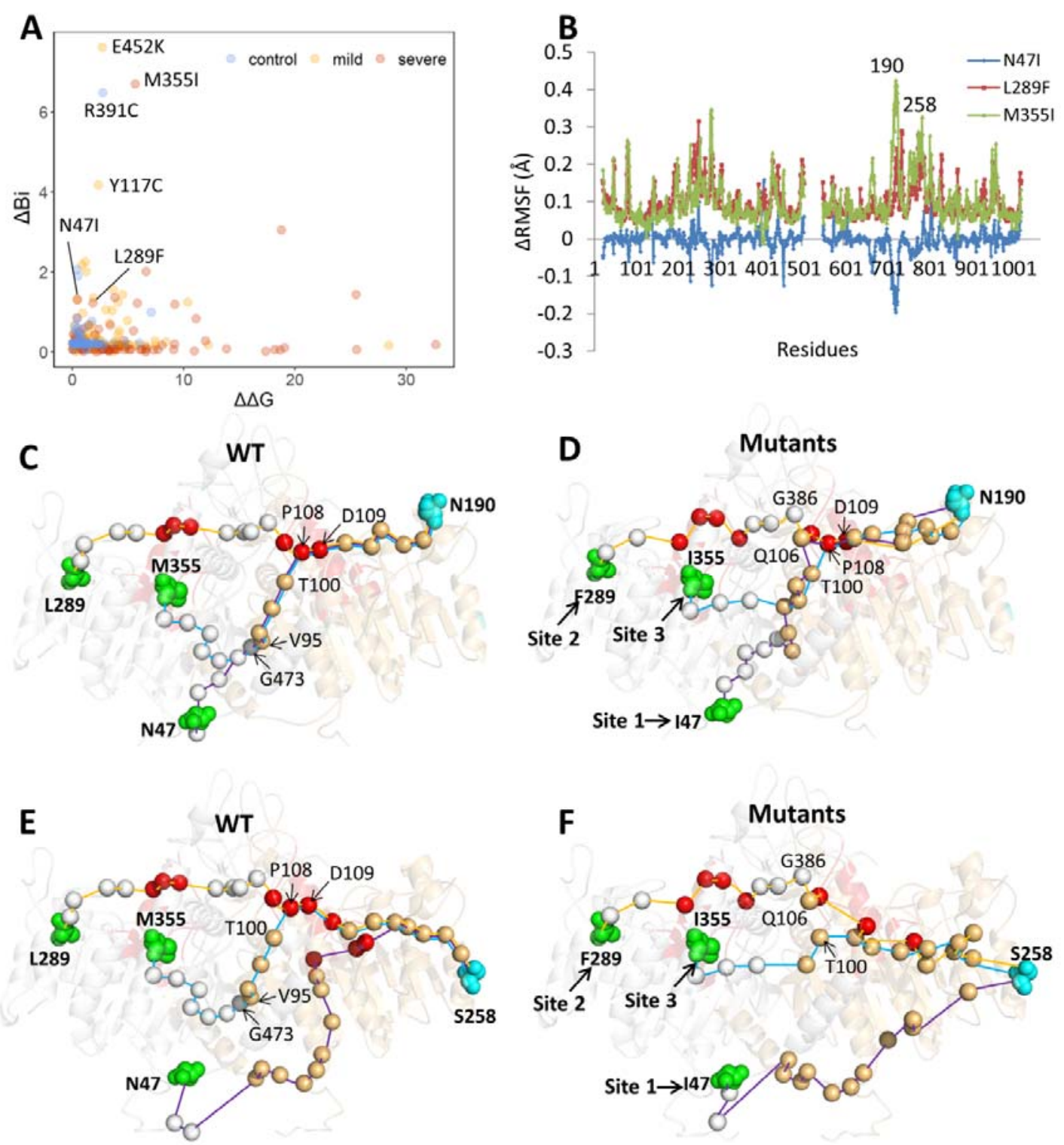

Fig 8. (A) The distribution of $\triangle \triangle \mathrm{G}$ and $\triangle \mathrm{B}$ for $A L P L$ mutations. The scatterplot showing distribution of $\Delta \mathrm{B}$ vs $\Delta \Delta \mathrm{G}$ of different mutation types. Severe, mild and control mutations are depicted in red, yellow, and blue, respectively. N47I, L289F, and M355I are three severe mutations with low $\Delta \Delta \mathrm{G}$ and high $\Delta \mathrm{B}$. Among the three significant mutations predicted by the scatterplot, two (E452K and R391K) were not originally included in the severe mutation group but were validated as two severe mutations in the newly collected clinical samples. (B) Differential RMSF ( $\triangle$ RMSF) of N47I (blue), L289F (red), and M355I (green), with respect to 
WT. Allosteric paths originating at three mutational sites and terminating at N190 in the WT (C) and mutant states (D), as well as terminating at S258 in the WT (E) and mutant states (F), respectively. The TNSALP structure is depicted as represented by semi-transparent colored cartoon, and the starting and ending residues of all the paths are represented as green and cyan spheres, respectively. The alpha-carbon of the path through the residues is shown as silver (chain A) and orange (chain B) spheres, in which active sites are represented by red spheres.

The comparison of allosteric pathways reveals several important insights. First, by using the same starting mutational sites and ending points, the mutant TNSALPs exhibit shorter allosteric pathways, which is in accordance with our hypothesis that the mutants caused by severe mutations have higher allosteric propensities. The system with the largest reduction in signaling path nodes is the mutant caused by M355I, with has the highest $\Delta \mathrm{B}$ and a relatively small $\Delta \Delta \mathrm{G}$. In WT, the path from M355 to S258 passed through the nodes $\mathrm{F} 462 \rightarrow \mathrm{S} 463 \rightarrow \mathrm{L} 471 \rightarrow \mathrm{H} 472 \rightarrow \mathrm{G} 473$ on the dimeric interface of the chain with mutations and the active sites P108 and D109 in the other chain (Fig 8E). In contrast, none of these sites participated in the signaling paths in the mutants. Hence the whole length becomes shorter and straight (Fig 8F). The same situation occurs in the path from M355I to N190 (Fig 8C, D). Second, the pathways in mutants involve fewer active sites, interfacial residues, and mutational sites. This comparison further highlights these functional regions as hubs for long-range allosteric communication in WTs, while it also means that the functional role of these reduced nodes may be lost if a severe mutation occurs. The extreme situation is observed for N47I, in which the path from I47 to S258 does not pass through the active area 
and employs the shortest and most straight path. Lastly, the path patterns are more robust in WT than in mutant states, suggesting that severe mutations introduce more pathway plasticity. For example, the shortest pathways from residues N47, L289, and M355 to N190 of the other chain in the WT shared some similar nodes, including P108, D109, G126, R184, D185, Y187, and S188, in the other chain, with most of located at the active site or ion binding pocket, and with all crossing the same area and to the ending residue N190 (orange circle region in Fig 8C and D). However, the situation was different in mutants. Fewer residues were shared in the three mutants, only residue P108 showed in the shortest pathway from I47, F289, and I355 to N190. The situation was similar in the paths from residues, I47, F289, and I355 to S258 of the other chain. Three paths shared residues, I204, A205, Y206, L252, and T255 of the other chain in the WT and none in the mutants.

Table 2. The constituent residues of the shortest pathway from the three severe mutational sites (N47, L289, and M355 to N190/S258). Residues in the two chains are denoted by different colors.

\begin{tabular}{|c|c|c|}
\hline Class & Start - end & Pathways \\
\hline \multirow{3}{*}{ WT } & N47 - N190 & $\begin{array}{c}\mathrm{T} 48 \rightarrow \mathrm{M} 467 \rightarrow \mathrm{L} 470 \rightarrow \mathrm{H} 472 \rightarrow \mathrm{V} 95 \rightarrow \mathrm{A} 96 \rightarrow \mathrm{S} 98 \rightarrow \mathrm{T} 100 \rightarrow \mathrm{P} 108 \rightarrow \mathrm{D} 109 \rightarrow \mathrm{G} 1 \\
26 \rightarrow \mathrm{R} 184 \rightarrow \mathrm{D} 185 \rightarrow \mathrm{Y} 187 \rightarrow \mathrm{S} 188\end{array}$ \\
\hline & L289 - N190 & $\begin{array}{l}\mathrm{F} 290 \rightarrow \mathrm{E} 291 \rightarrow \mathrm{Q} 296 \rightarrow \mathrm{Y} 297 \rightarrow \mathrm{H} 338 \rightarrow \mathrm{G} 339 \rightarrow \mathrm{H} 340 \rightarrow \mathrm{F} 383 \rightarrow \mathrm{T} 384 \rightarrow \mathrm{F} 385 \\
\quad \rightarrow \mathrm{G} 386 \rightarrow \mathrm{V} 107 \rightarrow \mathrm{P} 108 \rightarrow \mathrm{D} 109 \rightarrow \mathrm{G} 126 \rightarrow \mathrm{R} 184 \rightarrow \mathrm{D} 185 \rightarrow \mathrm{Y} 187 \rightarrow \mathrm{S} 188\end{array}$ \\
\hline & M355 - N190 & 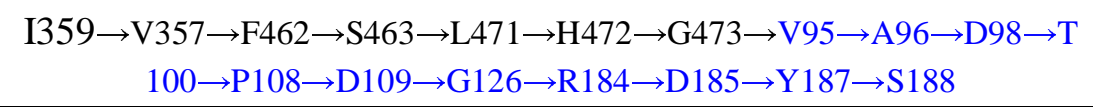 \\
\hline \multirow{3}{*}{ Mutants } & I47 - N190 & $\begin{aligned} \mathrm{M} 467 \rightarrow \mathrm{L} 470 \rightarrow \mathrm{L} 471 \rightarrow \mathrm{H} 472 \rightarrow \mathrm{G} 473 \rightarrow \mathrm{F} 94 \rightarrow \mathrm{V} 95 \rightarrow \mathrm{L} 97 \rightarrow \mathrm{S} 98 \rightarrow \mathrm{K} 99 \rightarrow \mathrm{V} 1 \\
07 \rightarrow \mathrm{P} 108 \rightarrow \mathrm{E} 125 \rightarrow \mathrm{A} 182 \rightarrow \mathrm{D} 185\end{aligned}$ \\
\hline & F289 - N190 & $\begin{array}{c}\mathrm{F} 290 \rightarrow \mathrm{M} 295 \rightarrow \mathrm{R} 335 \rightarrow \mathrm{G} 339 \rightarrow \mathrm{H} 340 \rightarrow \mathrm{H} 381 \rightarrow \mathrm{F} 383 \rightarrow \mathrm{T} 384 \rightarrow \mathrm{G} 386 \rightarrow \mathrm{Q} 106 \\
\rightarrow \mathrm{V} 107 \rightarrow \mathrm{P} 108 \rightarrow \mathrm{E} 125 \rightarrow \mathrm{A} 182 \rightarrow \mathrm{D} 185\end{array}$ \\
\hline & I355 - N190 & $\begin{aligned} & \mathrm{I} 359 \rightarrow \mathrm{V} 375 \rightarrow \mathrm{T} 176 \rightarrow \mathrm{A} 460 \rightarrow \mathrm{L} 97 \rightarrow \mathrm{S} 98 \rightarrow \mathrm{T} 100 \rightarrow \mathrm{P} 108 \rightarrow \mathrm{D} 109 \rightarrow \mathrm{G} 126 \rightarrow \mathrm{D} \\
& 183 \rightarrow \mathrm{W} 186 \rightarrow \mathrm{Y} 187 \rightarrow \mathrm{S} 188\end{aligned}$ \\
\hline WT & N47 - S258 & $\begin{aligned} \mathrm{P} 519 \rightarrow \mathrm{L} 523 & \rightarrow \mathrm{K} 45 \rightarrow \mathrm{L} 46 \rightarrow \mathrm{N} 47 \rightarrow \mathrm{N} 49 \rightarrow \mathrm{V} 50 \rightarrow \mathrm{A} 51 \rightarrow \mathrm{V} 54 \rightarrow \mathrm{M} 56 \rightarrow \mathrm{F} 57 \rightarrow \\
\mathrm{L} 58 & \rightarrow \mathrm{P} 174 \rightarrow \mathrm{S} 175 \rightarrow \mathrm{I} 204 \rightarrow \mathrm{A} 205 \rightarrow \mathrm{Y} 206 \rightarrow \mathrm{L} 252 \rightarrow \mathrm{T} 255\end{aligned}$ \\
\hline
\end{tabular}




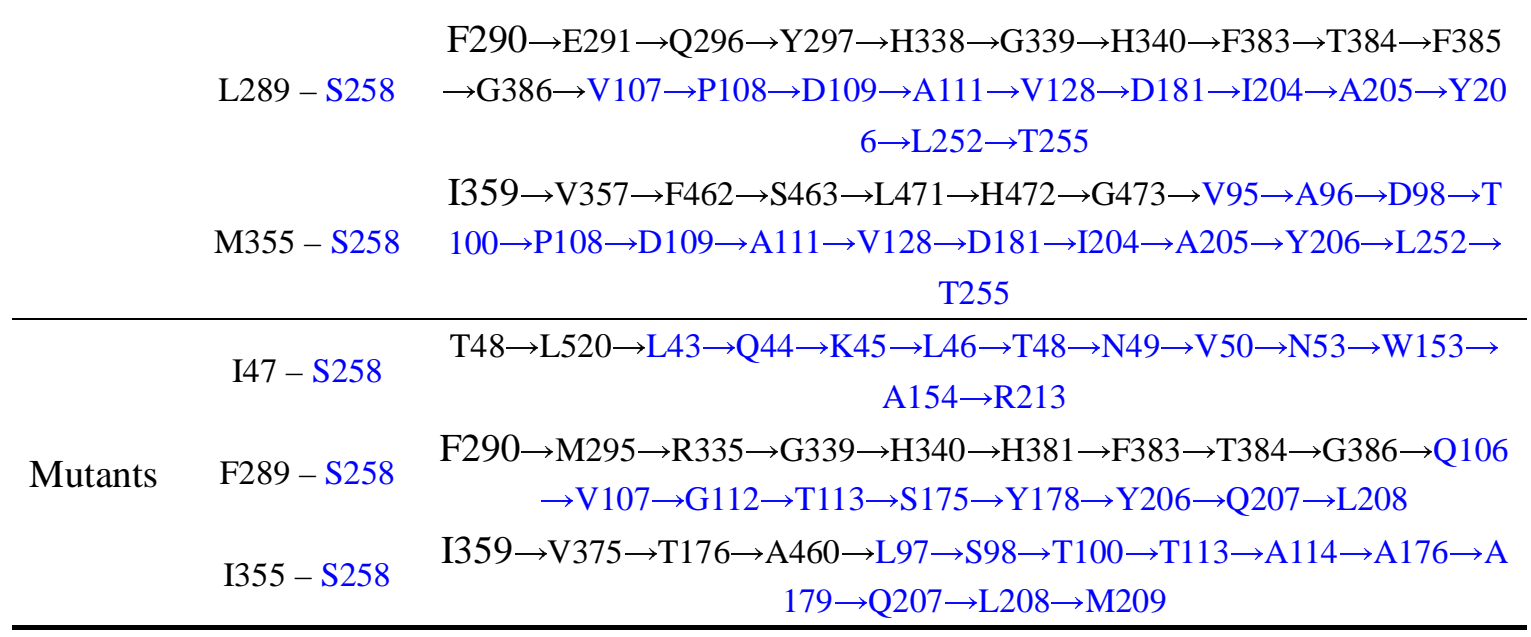

Taken together, the results suggeted that severe $A L P L$ mutaions may affect the pathway of signal transduction between the two monomers of TNSALP. Allosteric pathways in WTs are robust and involve some functionl sites, consisting of active, interfacial, and mutational residues. In the allosteric mutant states, the overall pathway pattern is more "flexible" with the shorter pathways involving fewer functional sites, resulting in the severe phenotype.

\section{Assessment of Allosteric Effects of Severe Mutational Sites}

As shown in Fig 8 (A), among the four mutations (E451K, M355I, R391C andY117C) with largest $\Delta \mathrm{B}$ but low $\Delta \Delta \mathrm{G}$ values, only M355I related to severe HPP phenotype. At the time of waiting, the ALPL mutation database has been updated by including several more severe mutations. We were surprised to find that the new patients with severe phenotype related to E452K and R391C, were reported as mild and control mutations previously. To further investigate their allosteric effects and whether is an intrinsic dynamic of these severe mutational sites, the single-residue perturbation analysis was performed by using the 
AlloSigMA. The results showed that perturbations of all these five mutational sites induced inter molecular allosteric effects (Fig 9) in the form of both positive and negative modulation with a mean value of $\Delta \mathrm{G}(\mathrm{N} 47 \rightarrow$ chain $\mathrm{B})=0.89 \mathrm{kcal} / \mathrm{mol}, \Delta \mathrm{G}(\mathrm{N} 289 \rightarrow$ chain $\mathrm{B})=0.11$ $\mathrm{kcal} / \mathrm{mol}, \Delta \mathrm{G}(\mathrm{N} 335 \rightarrow$ chain $\mathrm{B})=0.37 \mathrm{kcal} / \mathrm{mol}, \Delta \mathrm{G}(\mathrm{R} 391 \rightarrow$ chain $\mathrm{B})=0.13 \mathrm{kcal} / \mathrm{mol}$ and $\Delta \mathrm{G}(\mathrm{E} 452 \rightarrow$ chain $\mathrm{B})=0.08 \mathrm{kcal} / \mathrm{mol}$, respectively. The analysis exhibited an evident influence on the stability of some functional regions, including the $\mathrm{Ca}^{2+}$ binding and crown domain, thereby revealing that severe mutations can induce changes in the stability of other sites, and affect the catalytic activity of proteins.

A

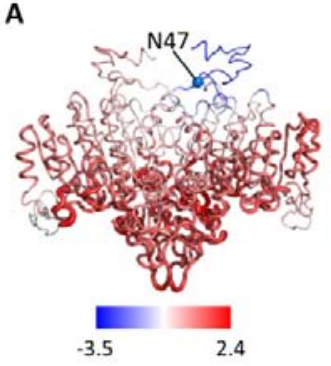

E

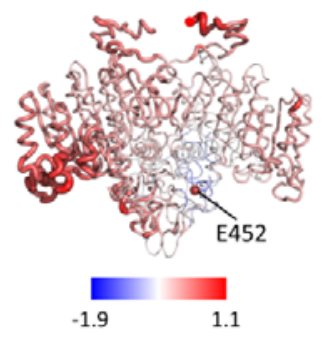

B

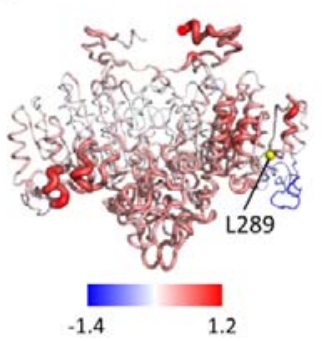

C

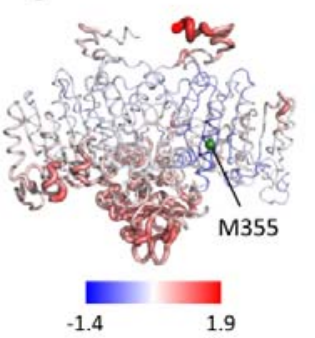

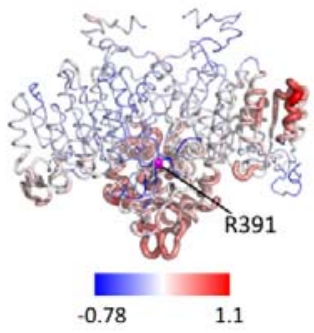

$\mathbf{F}$

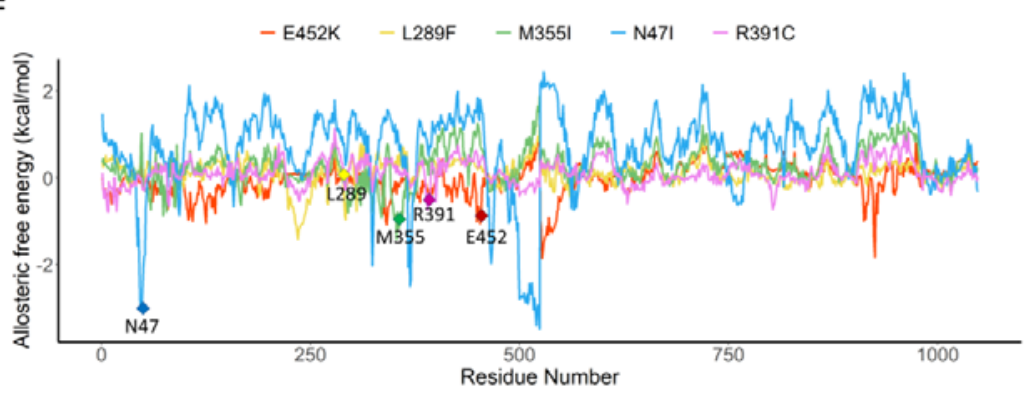

Fig 9. Allosteric effects of three studied severe mutations (N47I, L289F and M355I) and two predicted severe mutations (R391C and E452K) calculated by AlloSigMA. Cartoon structures of the TNSALP protein colored according to their free energy values obtained for the cases of (A) N47I, (B) L289F, (C) M355I, (D) R391C and (E) E452K, while blue color indicates negative allosteric free energy and red color indicates positive modulation. $(\mathrm{F})$ Their 
free energy profiles are illustrated graphically with residue index (chain A: 1-524; chain B: 525-1048) on the $\mathrm{x}$-axis and $\Delta \mathrm{g}$ value on the $\mathrm{y}$-axis. Blue, yellow, green, pink and red profiles represent the result for N47I, L289F, M355I, R391C and E452K respectively.

These findings once again suggest that $\Delta \mathrm{B}$ is a good and facile indictor for predicting severe mutations, whose molecular pathogenicity does not alter the local stability at active sites so as to change the protein folding energy, but instead generates alternative and long-range molecular effects.

\section{Conclusions}

As the biological hallmark of HPP, the reduced activity of TNSALP is caused by loss-of-function mutations in the $A L P L$ gene; varying levels of reduced activity are related to mild or severe HPP phenotypes. Despite some progress, more reseach is needed for comprehensive understanding of genotype-phenotype interrelationship in HPP. Thus, in this study, the functional landscape of $A L P L$ mutations was established by investigating a set of features generated from the protein sequence, network topology, and ENM dynamics calculation, and their relationship with different HPP phenotypes. Based on these features, a machine learning classifier was developed not only to examine the relationship between the pathogenicity status of mutations and their biophysical attributes, but also for the prediction of mutations in the control, mild, and severe groups. We have found that in addition to $\Delta \Delta \mathrm{G}$ as the commonly used predictor, co-evolutionary conservation and network-based features also yield strong signals in machine learning predictions and provide orthogonal information. 
Further structure-function studies including MD simulation and structural-communication pathway analysis of mutant TNSALPs, have supported our arguments that mutational hotspot sites often correspond to global mediators of allosteric interactions. These findings suggest that the study of molecular signatures and allosteric regulation of $A L P L$ mutations may be a step toward defining a greater quantitative genotype-phenotype interrelationship in HPP.

Through the large-scale analysis of disease-causing ALPL mutations, we propose the following possible molecular principles underlying HPP-related mutations. First, HPP pathogenicity is largely due to the structural instability of the TNSALP caused by $A L P L$ mutations, which have variable effects on enzyme activity. Thus, for such cases, $\Delta \Delta \mathrm{G}$ is a satisfactory and acceptable index for predicting pathogenic mutations, especially for distinguishing mutations between the control and severe groups. There is also the possibility of extrapolating our methods of using $\Delta \Delta \mathrm{G}$ to estimate change in protein stability upon mutations as a popular way to predict pathogenic mutations in other diseases. Second, the most interesting finding in our opinion is that $\Delta \mathrm{B}$ has been predicted as a good network indicator to distinguish mild and severe groups in pathogenic mutations. We speculate that mutations in the severe group have stronger allosteric effects than mutations related to the milder forms of HPP, severing as "allosteric mutations".[78, 79] Analysis of allosteric properties of severe mutations adds an additional confirmatory layer to segregate variants in "mild" and "severe" states, thereby furthering understanding of the allosteric basis of loss-of-function mutations. Third, for the classification of mild mutations and differentiation from the control group, co-evolutionary conservation has been shown to be the most important predictor, thereby suggesting that mild pathogenicity may be related to amino acid 
changes with small evolutionary substitution probability. However, to reach the elusive goal of establishing the precise relationship between $A L P L$ mutation genotypes and HPP phenotypes and a more reliable prediction model or score, like as protein regulatory and functional binding sites prediction done,[80] more clinical data on mutations[81] and data on enzyme activity[13] are needed.

\section{Supporting Information}

S1 Table. Collected data set of ALPL mutations.

S1 Fig. Distribution of ALPL mutations in terms of WT TNSALP sequence and structure.

S2 Fig. Evolutionary conservation analysis performed for TNSALP protein P60484 with 403 aa using ConSurf.

S3 Fig. Ramachandran plot of TNSALP.

S4 Fig. Comparisons of different molecular signatures for ALPL mutations.

S5 Fig. Conformational dynamics of TNSALPs.

\section{Acknowledgments}

This work was supported by the National Natural Science Foundation of China (31872723), a Project Funded by the Priority Academic Program Development (PAPD) of Jiangsu Higher Education Institutions, the China Postdoctoral Science Foundation (2016M590495), and the Jiangsu Planned Projects for Postdoctoral Research Funds (1601168C). 


\section{Author Contributions}

The manuscript was written through contributions of all authors. All authors have given approval to the final version of the manuscript. $\ddagger$ These authors contributed equally.

Notes

The authors declare no competing financial interest.

\section{Abbreviations}

HPP, Hypophosphatasia; TNSALP, tissue-non-specific alkaline phosphatase; MSA, Multiple Sequence Alignment; SASA, solvent-accessible surface area; PSN, protein structure network; ENM, elastic network model; RF, Random Forest; ROC, Receiver Operating Characteristics; AUC, Area Under Curve.

\section{References}

1. Rathbun JC. Hypophosphatasia; a new developmental anomaly. American journal of diseases of children. 1948;75(6):822-831. doi: 10.1001/archpedi.1948.02030020840003

2. Mornet E. Hypophosphatasia. Metabolism: clinical and experimental. 2018;82:142-155. doi: 10.1016/j.metabol.2017.08.013

3. Komaru K, Ishida-Okumura Y, Numa-Kinjoh N, Hasegawa T, Oda K. Molecular and cellular basis of hypophosphatasia. Journal of oral biosciences. 2019;61(3):141-148. doi: 10.1016/j.job.2019.07.003

4. Whyte MP, Zhang F, Wenkert D, McAlister WH, Mack KE, Benigno MC, et al. Hypophosphatasia: validation and expansion of the clinical nosology for children from 25 years experience with 173 pediatric patients. Bone. 2015;75:229-239. doi: 10.1016/j.bone.2015.02.022

5. Vimalraj S. Alkaline phosphatase: Structure, expression and its function in bone mineralization. Gene. 2020;754:144855. doi: 10.1016/j.gene.2020.144855

6. Millan JL, Whyte MP. Alkaline Phosphatase and Hypophosphatasia. Calcified tissue international. 2016;98(4):398-416. doi: 10.1007/s00223-015-0079-1

7. Whyte MP, Greenberg CR, Salman NJ, Bober MB, McAlister WH, Wenkert D, et al. Enzyme-replacement 
therapy in life-threatening hypophosphatasia. The New England journal of medicine. 2012;366(10):904-913. doi: 10.1056/NEJMoa1106173

8. Bianchi ML, Vai S. Alkaline Phosphatase Replacement Therapy. Advances in experimental medicine and biology. 2019;1148:201-232. doi: 10.1007/978-981-13-7709-9_10

9. Kyostila K, Syrja P, Lappalainen AK, Arumilli M, Hundi S, Karkamo V, et al. A homozygous missense variant in the alkaline phosphatase gene ALPL is associated with a severe form of canine hypophosphatasia. Sci Rep-Uk. 2019;9. doi: 10.1038/s41598-018-37801-2

10. Bianchi ML. Hypophosphatasia: an overview of the disease and its treatment. Osteoporosis international : a journal established as result of cooperation between the European Foundation for Osteoporosis and the National Osteoporosis Foundation of the USA. 2015;26(12):2743-2757. doi: 10.1007/s00198-015-3272-1

11. Whyte MP. Hypophosphatasia - aetiology, nosology, pathogenesis, diagnosis and treatment. Nature reviews Endocrinology. 2016;12(4):233-246. doi: 10.1038/nrendo.2016.14

12. Silvent J, Gasse B, Mornet E, Sire JY. Molecular evolution of the tissue-nonspecific alkaline phosphatase allows prediction and validation of missense mutations responsible for hypophosphatasia. The Journal of biological chemistry. 2014;289(35):24168-24179. doi: 10.1074/jbc.M114.576843

13. del Angel G, Reynders J, Negron C, Steinbrecher T, Mornet E. Large-scale in vitro functional testing and novel variant scoring via protein modeling provide insights into alkaline phosphatase activity in hypophosphatasia. Hum Mutat. 2020. doi: 10.1002/humu.24010

14. Mornet E, Taillandier A, Domingues C, Dufour A, Benaloun E, Lavaud N, et al. Hypophosphatasia: a genetic-based nosology and new insights in genotype-phenotype correlation. European journal of human genetics : EJHG. 2020. doi: 10.1038/s41431-020-00732-6

15. Zaher DM, El-Gamal MI, Omar HA, Aljareh SN, Al-Shamma SA, Ali AJ, et al. Recent advances with alkaline phosphatase isoenzymes and their inhibitors. Archiv der Pharmazie. 2020;353(5):e2000011. doi: 10.1002/ardp.202000011

16. Mornet E, Stura E, Lia-Baldini AS, Stigbrand T, Menez A, Le Du MH. Structural evidence for a functional role of human tissue nonspecific alkaline phosphatase in bone mineralization. The Journal of biological chemistry. 2001;276(33):31171-31178. doi: 10.1074/jbc.M102788200

17. Numa N, Ishida $Y$, Nasu M, Sohda M, Misumi $Y$, Noda $T$, et al. Molecular basis of perinatal hypophosphatasia with tissue-nonspecific alkaline phosphatase bearing a conservative replacement of valine by alanine at position 406. Structural importance of the crown domain. The FEBS journal. 2008;275(11):2727-2737. doi: 10.1111/j.1742-4658.2008.06414.x

18. Hoylaerts MF, Manes T, Millan JL. Mammalian alkaline phosphatases are allosteric enzymes. The Journal of biological chemistry. 1997;272(36):22781-22787. doi: 10.1074/jbc.272.36.22781

19. Martins L, de Almeida AB, Dos Santos EJL, Foster BL, Machado RA, Kantovitz KR, et al. A novel combination of biallelic ALPL mutations associated with adult hypophosphatasia: A phenotype-genotype association and computational analysis study. Bone. 2019;125:128-139. doi: 10.1016/j.bone.2019.05.005

20. Borges B, Gallo G, Coelho C, Negri N, Maiello F, Hardy L, et al. Dynamic cross correlation analysis of Thermus thermophilus alkaline phosphatase and determinants of thermostability. Biochimica et biophysica acta General subjects. 2021;1865(7):129895. doi: 10.1016/j.bbagen.2021.129895

21. Li Y, Song K, Zhang J, Lu SY. A Computational Method to Predict Effects of Residue Mutations on the Catalytic Efficiency of Hydrolases. Catalysts. 2021;11(2). doi: 10.3390/Catal11020286

22. Tang N, Sandahl TD, Ott P, Kepp KP. Computing the Pathogenicity of Wilson's Disease ATP7B Mutations: Implications for Disease Prevalence. J Chem Inf Model. 2019;59(12):5230-5243. doi: 10.1021/acs.jcim.9b00852

23. Cheng $\mathrm{N}$, Li M, Zhao L, Zhang B, Yang $\mathrm{Y}$, Zheng $\mathrm{CH}$, et al. Comparison and integration of computational 
methods for deleterious synonymous mutation prediction. Briefings in bioinformatics. 2020;21(3):970-981. doi: 10.1093/bib/bbz047

24. Li G, Pahari S, Krishna Murthy A, Liang S, Fragoza R, Yu H, et al. SAAMBE-SEQ: A Sequence-based Method for Predicting Mutation Effect on Protein-protein Binding Affinity. Bioinformatics. 2020. doi: 10.1093/bioinformatics/btaa761

25. Kucukkal TG, Petukh M, Li L, Alexov E. Structural and physico-chemical effects of disease and non-disease nsSNPs on proteins. Current opinion in structural biology. 2015;32:18-24. doi: 10.1016/j.sbi.2015.01.003

26. Ghosh A, Vishveshwara S. A study of communication pathways in methionyl- tRNA synthetase by molecular dynamics simulations and structure network analysis. Proceedings of the National Academy of Sciences of the United States of America. 2007;104(40):15711-15716. doi: 10.1073/pnas.0704459104

27. Sirithep K, Xiao F, Raethong N, Zhang Y, Laoteng K, Hu G, et al. Probing Carbon Utilization of Cordyceps militaris by Sugar Transportome and Protein Structural Analysis. Cells. 2020;9(2). doi: 10.3390/cells9020401

28. Brinda KV, Vishveshwara S. A network representation of protein structures: implications for protein stability. Biophysical journal. 2005;89(6):4159-4170. doi: 10.1529/biophysj.105.064485

29. Achoch M, Dorantes-Gilardi R, Wymant C, Feverati G, Salamatian K, Vuillon L, et al. Protein structural robustness to mutations: an in silico investigation. Physical chemistry chemical physics : PCCP. 2016;18(20):13770-13780. doi: 10.1039/c5cp06091e

30. Giollo M, Martin AJ, Walsh I, Ferrari C, Tosatto SC. NeEMO: a method using residue interaction networks to improve prediction of protein stability upon mutation. BMC genomics. 2014;15 Suppl 4:S7. doi: 10.1186/1471-2164-15-S4-S7

31. Kumar A, Glembo TJ, Ozkan SB. The Role of Conformational Dynamics and Allostery in the Disease Development of Human Ferritin. Biophysical journal. 2015;109(6):1273-1281. doi: 10.1016/j.bpj.2015.06.060

32. Atilgan AR, Durell SR, Jernigan RL, Demirel MC, Keskin O, Bahar I. Anisotropy of fluctuation dynamics of proteins with an elastic network model. Biophysical journal. 2001;80(1):505-515. doi: 10.1016/S0006-3495(01)76033-X

33. Bahar I, Atilgan AR, Erman B. Direct evaluation of thermal fluctuations in proteins using a single-parameter harmonic potential. Folding \& design. 1997;2(3):173-181. doi: 10.1016/S1359-0278(97)00024-2

34. Frappier V, Najmanovich RJ. A coarse-grained elastic network atom contact model and its use in the simulation of protein dynamics and the prediction of the effect of mutations. PLoS computational biology. 2014;10(4):e1003569. doi: 10.1371/journal.pcbi.1003569

35. Sayilgan JF, Haliloglu T, Gonen M. Protein dynamics analysis reveals that missense mutations in cancer-related genes appear frequently on hinge-neighboring residues. Proteins. 2019;87(6):512-519. doi: 10.1002/prot. 25673

36. Ponzoni L, Bahar I. Structural dynamics is a determinant of the functional significance of missense variants. Proceedings of the National Academy of Sciences of the United States of America. 2018;115(16):4164-4169. doi: 10.1073/pnas.1715896115

37. Agajanian S, Odeyemi O, Bischoff N, Ratra S, Verkhivker GM. Machine Learning Classification and Structure-Functional Analysis of Cancer Mutations Reveal Unique Dynamic and Network Signatures of Driver Sites in Oncogenes and Tumor Suppressor Genes. J Chem Inf Model. 2018;58(10):2131-2150. doi: 10.1021/acs.jcim.8b00414

38. Agajanian S, Oluyemi O, Verkhivker GM. Integration of Random Forest Classifiers and Deep Convolutional Neural Networks for Classification and Biomolecular Modeling of Cancer Driver Mutations. Frontiers in molecular biosciences. 2019;6:44. doi: 10.3389/fmolb.2019.00044

39. Verkhivker GM, Agajanian S, Hu G, Tao P. Allosteric Regulation at the Crossroads of New Technologies: 
Multiscale Modeling, Networks, and Machine Learning. Frontiers in molecular biosciences. 2020;7:136. doi: 10.3389/fmolb.2020.00136

40. Verkhivker GM. Biophysical simulations and structure-based modeling of residue interaction networks in the tumor suppressor proteins reveal functional role of cancer mutation hotspots in molecular communication. Biochimica et biophysica acta General subjects. 2019;1863(1):210-225. doi: 10.1016/j.bbagen.2018.10.009 41. Stetz G, Astl L, Verkhivker GM. Exploring Mechanisms of Communication Switching in the Hsp90-Cdc37 Regulatory Complexes with Client Kinases through Allosteric Coupling of Phosphorylation Sites: Perturbation-Based Modeling and Hierarchical Community Analysis of Residue Interaction Networks. Journal of chemical theory and computation. 2020;16(7):4706-4725. doi: 10.1021/acs.jctc.0c00280

42. Verkhivker G, Agajanian S, Oztas D, Gupta G. Dynamic Profiling of Binding and Allosteric Propensities of the SARS-CoV-2 Spike Protein with Different Classes of Antibodies: Mutational and Perturbation-Based Scanning Reveals the Allosteric Duality of Functionally Adaptable Hotspots. Journal of chemical theory and computation. 2021;17(7):4578-4598. doi: 10.1021/acs.jctc.1c00372

43. Smith IN, Thacker S, Seyfi M, Cheng F, Eng C. Conformational Dynamics and Allosteric Regulation Landscapes of Germline PTEN Mutations Associated with Autism Compared to Those Associated with Cancer. American journal of human genetics. 2019;104(5):861-878. doi: 10.1016/j.ajhg.2019.03.009

44. Portelli S, Barr L, de Sa AGC, Pires DEV, Ascher DB. Distinguishing between PTEN clinical phenotypes through mutation analysis. Comput Struct Biotec. 2021;19:3097-3109. doi: 10.1016/j.csbj.2021.05.028

45. Murthy ASN, Suresh RV, Nallur BR. Comprehensive in silico mutational-sensitivity analysis of PTEN establishes signature regions implicated in pathogenesis of Autism Spectrum Disorders. Genomics. 2021;113(1):999-1017. doi: 10.1016/j.ygeno.2020.10.035

46. Liang ZJ, Verkhivker GM, Hu G. Integration of network models and evolutionary analysis into high-throughput modeling of protein dynamics and allosteric regulation: theory, tools and applications. Briefings in bioinformatics. 2020;21(3):815-835. doi: 10.1093/bib/bbz029

47. Astl L, Verkhivker GM. Data-driven computational analysis of allosteric proteins by exploring protein dynamics, residue coevolution and residue interaction networks. Biochimica et biophysica acta General subjects. 2019. doi: 10.1016/j.bbagen.2019.07.008

48. Liang Z, Hu J, Yan W, Jiang H, Hu G, Luo C. Deciphering the role of dimer interface in intrinsic dynamics and allosteric pathways underlying the functional transformation of DNMT3A. Biochimica et biophysica acta General subjects. 2018;1862(7):1667-1679. doi: 10.1016/j.bbagen.2018.04.015

49. Xiao F, Song X, Tian P, Gan M, Verkhivker GM, Hu G. Comparative Dynamics and Functional Mechanisms of the CYP17A1 Tunnels Regulated by Ligand Binding. J Chem Inf Model. 2020;60(7):3632-3647. doi: 10.1021/acs.jcim.0c00447

50. Iqbal S, Perez-Palma E, Jespersen JB, May P, Hoksza D, Heyne HO, et al. Comprehensive characterization of amino acid positions in protein structures reveals molecular effect of missense variants. Proceedings of the National Academy of Sciences of the United States of America. 2020;117(45):28201-28211. doi: 10.1073/pnas.2002660117

51. Tack DS, Tonner PD, Pressman A, Olson ND, Levy SF, Romantseva EF, et al. The genotype-phenotype landscape of an allosteric protein. Molecular systems biology. 2021;17(3):e10179. doi: 10.15252/msb.202010179

52. Ashkenazy H, Abadi S, Martz E, Chay O, Mayrose I, Pupko T, et al. ConSurf 2016: an improved methodology to estimate and visualize evolutionary conservation in macromolecules. Nucleic Acids Res. 2016;44(W1):W344-350. doi: 10.1093/nar/gkw408

53. Bakan A, Dutta A, Mao W, Liu Y, Chennubhotla C, Lezon TR, et al. Evol and ProDy for bridging protein 
sequence evolution and structural dynamics. Bioinformatics. 2014;30(18):2681-2683. doi: 10.1093/bioinformatics/btu336

54. Marti-Renom MA, Stuart AC, Fiser A, Sanchez R, Melo F, Sali A. Comparative protein structure modeling of genes and genomes. Annual review of biophysics and biomolecular structure. 2000;29:291-325. doi: 10.1146/annurev.biophys.29.1.291

55. Llinas P, Stura EA, Menez A, Kiss Z, Stigbrand T, Millan JL, et al. Structural studies of human placental alkaline phosphatase in complex with functional ligands. Journal of molecular biology. 2005;350(3):441-451. doi: 10.1016/j.jmb.2005.04.068

56. Zhang Z, Wang L, Gao Y, Zhang J, Zhenirovskyy M, Alexov E. Predicting folding free energy changes upon single point mutations. Bioinformatics. 2012;28(5):664-671. doi: 10.1093/bioinformatics/bts005

57. Besterman AD, Althoff T, Elfferich P, Gutierrez-Mejia I, Sadik J, Bernstein JA, et al. Functional and structural analyses of novel Smith-Kingsmore Syndrome-Associated MTOR variants reveal potential new mechanisms and predictors of pathogenicity. PLoS genetics. 2021;17(7):e1009651. doi: 10.1371/journal.pgen.1009651

58. Mihel J, Sikic M, Tomic S, Jeren B, Vlahovicek K. PSAIA - Protein structure and interaction analyzer. Bmc Struct Biol. 2008;8. doi: 10.1186/1472-6807-8-21

59. Yan W, Hu G, Liang Z, Zhou J, Yang Y, Chen J, et al. Node-Weighted Amino Acid Network Strategy for Characterization and Identification of Protein Functional Residues. J Chem Inf Model. 2018;58(9):2024-2032. doi: 10.1021/acs.jcim.8b00146

60. Yan W, Sun M, Hu G, Zhou J, Zhang W, Chen J, et al. Amino acid contact energy networks impact protein structure and evolution. Journal of theoretical biology. 2014;355:95-104. doi: 10.1016/j.jtbi.2014.03.032

61. Doncheva NT, Klein K, Domingues FS, Albrecht M. Analyzing and visualizing residue networks of protein structures. Trends in biochemical sciences. 2011;36(4):179-182. doi: 10.1016/j.tibs.2011.01.002

62. Bakan A, Meireles LM, Bahar I. ProDy: protein dynamics inferred from theory and experiments. Bioinformatics. 2011;27(11):1575-1582. doi: 10.1093/bioinformatics/btr168

63. Atilgan $C$, Atilgan AR. Perturbation-response scanning reveals ligand entry-exit mechanisms of ferric binding protein. PLoS computational biology. 2009;5(10):e1000544. doi: 10.1371/journal.pcbi.1000544

64. Tyner S, Briatte F, Hofmann H. Network Visualization with ggplot2. R J. 2017;9(1):27-59.

65. Van Der Spoel D, Lindahl E, Hess B, Groenhof G, Mark AE, Berendsen HJ. GROMACS: fast, flexible, and free. Journal of computational chemistry. 2005;26(16):1701-1718. doi: 10.1002/jcc.20291

66. Lindorff-Larsen K, Piana S, Palmo K, Maragakis P, Klepeis JL, Dror RO, et al. Improved side-chain torsion potentials for the Amber ff99SB protein force field. Proteins. 2010;78(8):1950-1958. doi: 10.1002/prot.22711

67. Essmann U, Perera L, Berkowitz ML, Darden T, Lee H, Pedersen LG. A Smooth Particle Mesh Ewald Method. J Chem Phys. 1995;103(19):8577-8593. doi: Doi 10.1063/1.470117

68. Hess B, Bekker H, Berendsen HJC, Fraaije JGEM. LINCS: A linear constraint solver for molecular simulations. Journal of computational chemistry. 1997;18(12):1463-1472. doi: Doi 10.1002/(Sici)1096-987x(199709)18:12<1463::Aid-Jcc4>3.3.Co;2-L

69. Bussi G, Donadio D, Parrinello M. Canonical sampling through velocity rescaling. J Chem Phys. 2007;126(1). doi: $10.1063 / 1.2408420$

70. Brown DK, Penkler DL, Amamuddy OS, Ross C, Atilgan AR, Atilgan C, et al. MD-TASK: a software suite for analyzing molecular dynamics trajectories. Bioinformatics. 2017;33(17):2768-2771. doi: 10.1093/bioinformatics/btx349

71. Floyd RW. Algorithm-97 - Shortest Path. Commun Acm. 1962;5(6):345-345. doi: Doi $10.1145 / 367766.368168$

72. Guarnera E, Tan ZW, Zheng Z, Berezovsky IN. AlloSigMA: allosteric signaling and mutation analysis server. 
Bioinformatics. 2017;33(24):3996-3998. doi: 10.1093/bioinformatics/btx430

73. Tan ZW, Guarnera E, Tee WV, Berezovsky IN. AlloSigMA 2: paving the way to designing allosteric effectors and to exploring allosteric effects of mutations. Nucleic Acids Res. 2020;48(W1):W116-W124. doi: 10.1093/nar/gkaa338

74. Hu G, Yan WY, Zhou JH, Shen BR. Residue interaction network analysis of Dronpa and a DNA clamp. Journal of theoretical biology. 2014;348:55-64. doi: 10.1016/j.jtbi.2014.01.023

75. Kapetis D, Sassone J, Yang Y, Galbardi B, Xenakis MN, Westra RL, et al. Network topology of NaV1.7 mutations in sodium channel-related painful disorders. BMC systems biology. 2017;11(1):28. doi: 10.1186/s12918-016-0382-0

76. Sayilgan JF, Haliloglu T, Gonen M. Protein dynamics analysis identifies candidate cancer driver genes and mutations in TCGA data. Proteins. 2021. doi: 10.1002/prot.26054

77. Leander $M$, Yuan $Y$, Meger A, Cui $Q$, Raman S. Functional plasticity and evolutionary adaptation of allosteric regulation. Proceedings of the National Academy of Sciences of the United States of America. 2020;117(41):25445-25454. doi: 10.1073/pnas.2002613117

78. Lu S, Qiu Y, Ni D, He X, Pu J, Zhang J. Emergence of allosteric drug-resistance mutations: new challenges for allosteric drug discovery. Drug discovery today. 2020;25(1):177-184. doi: 10.1016/j.drudis.2019.10.006

79. Tan ZW, Tee WV, Guarnera E, Booth L, Berezovsky IN. AlloMAPS: allosteric mutation analysis and polymorphism of signaling database. Nucleic Acids Research. 2019;47(D1):D265-D270. doi: 10.1093/nar/gky1028

80. Mishra SK, Kandoi G, Jernigan RL. Coupling dynamics and evolutionary information with structure to identify protein regulatory and functional binding sites. Proteins. 2019;87(10):850-868. doi: 10.1002/prot.25749

81. Farris J, Calhoun B, Alam MS, Lee S, Haldar K. Large scale analyses of genotype-phenotype relationships of glycine decarboxylase mutations and neurological disease severity. PLoS computational biology. 2020;16(5):e1007871. doi: 10.1371/journal.pcbi.1007871 
A

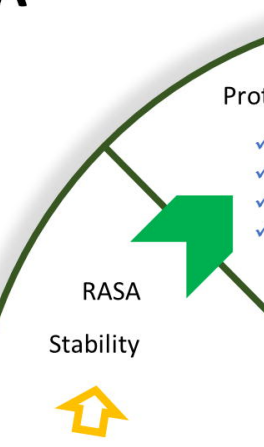

Structural Modeling

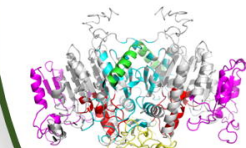

Protein Structure Network Centrality

degree

betweenness

$\checkmark$ closeness

clustering coefficient

\section{HGMD JKU PubMed}

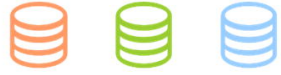

mutations

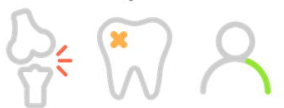
severe mild control

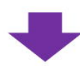

Sequence Conservation

1 S ELVIMAI GTCTrNSINVP

$f$

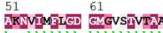

sff s s ss ss s s
s s s

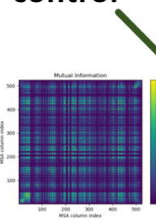

Coevolution

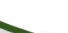

Dynamic Features

based on ENM

B

Large-scale analysis of mutations
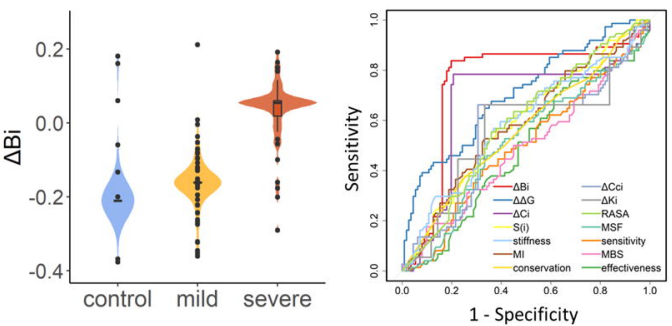

C

Allosteric effect analysis

$\checkmark$ MSF

$\checkmark$ effectiveness

$\checkmark$ sensitivity

$\checkmark$ MBS

$\checkmark$ stiffness xytum
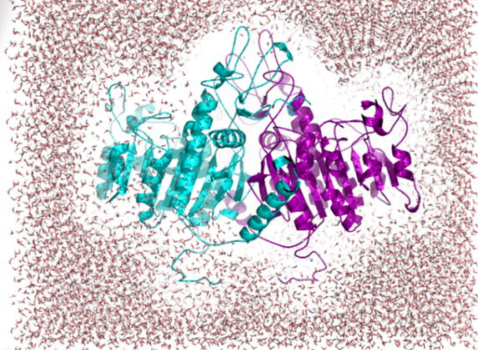

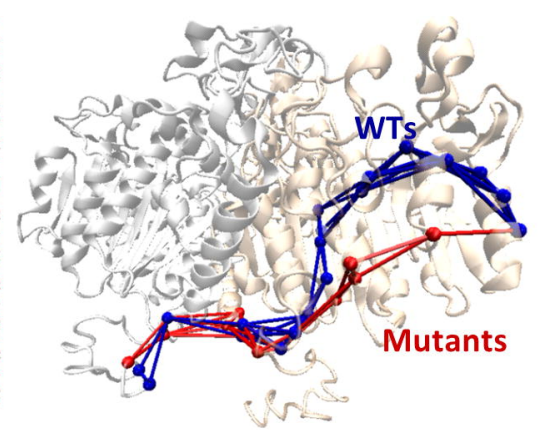


A
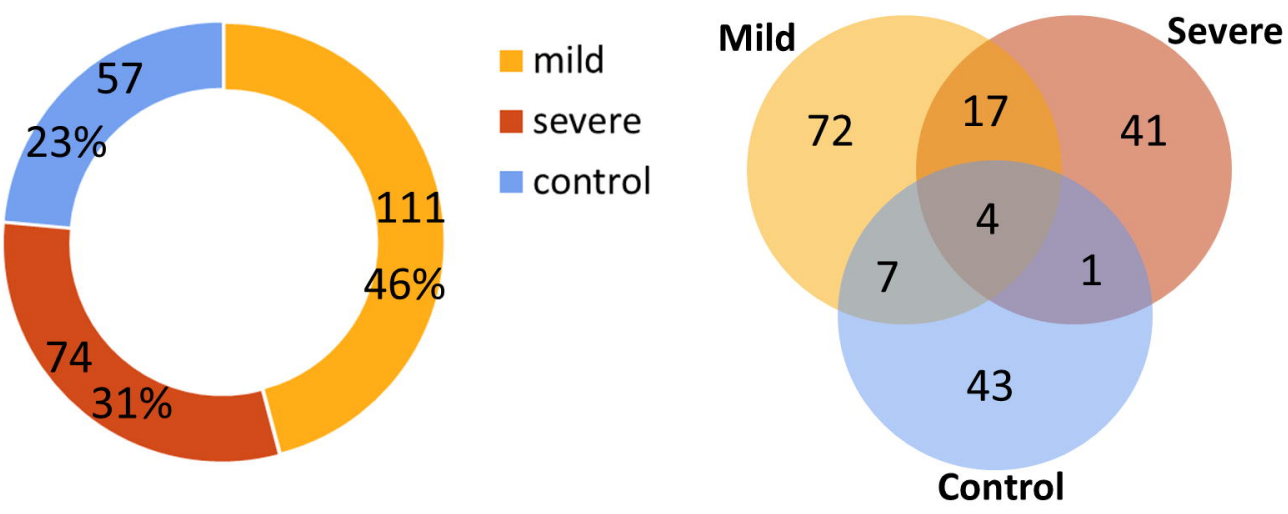

B

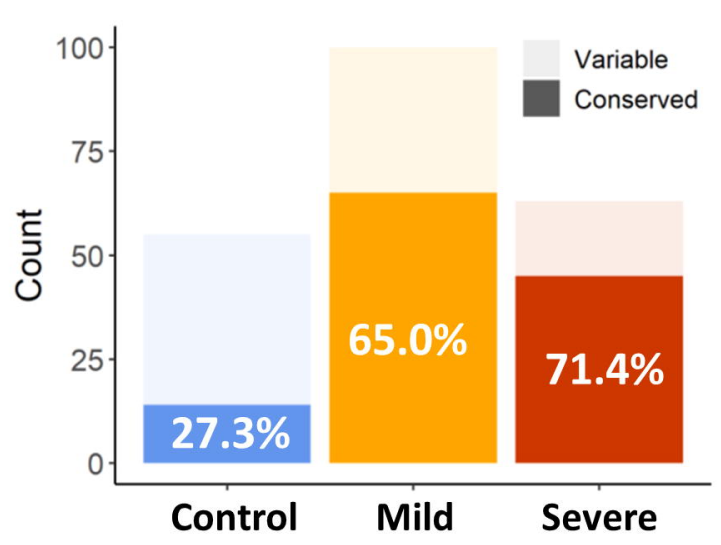

C

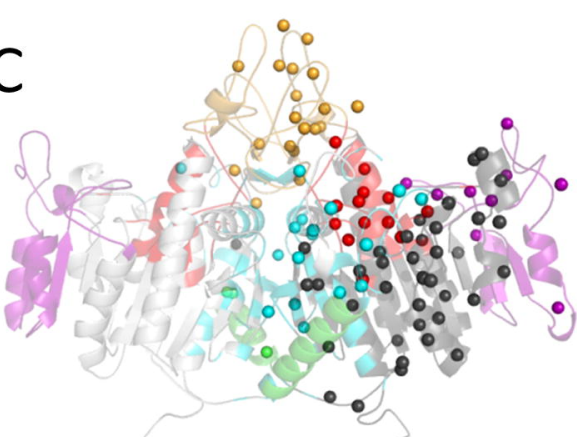

Mild

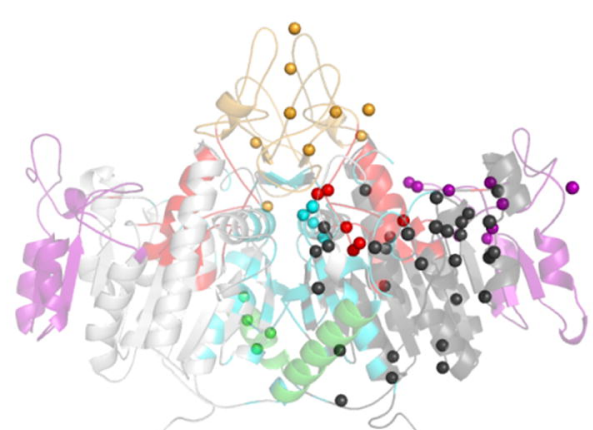

Severe

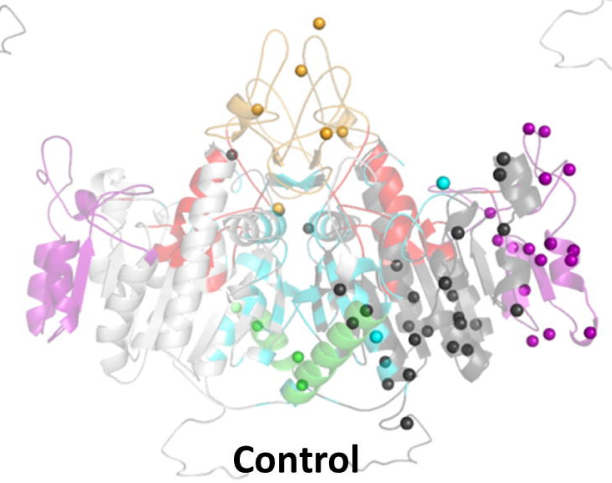

Control

\section{D}
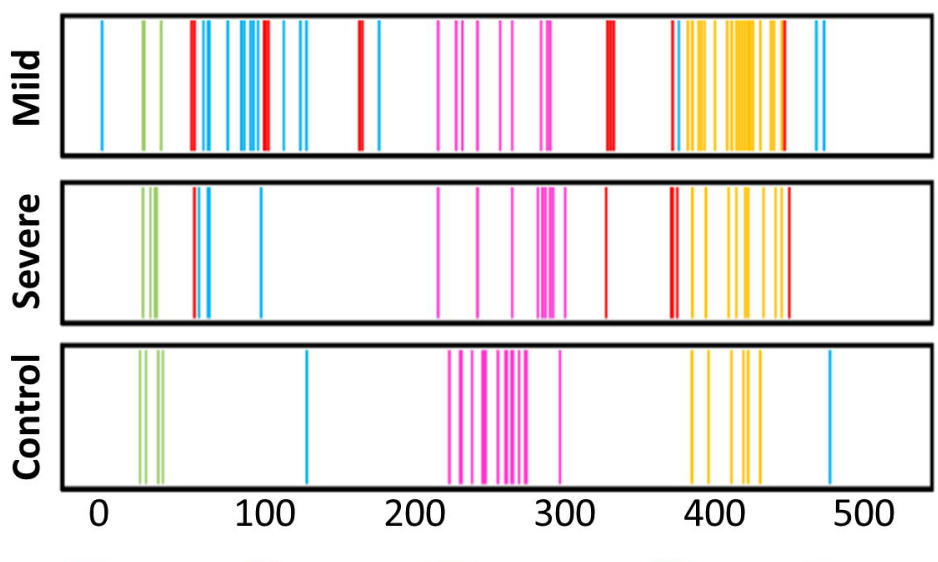

active site $\square$ calcium site $\square$ crown domain $\square$ interface $\square \mathrm{N}$-term 
A

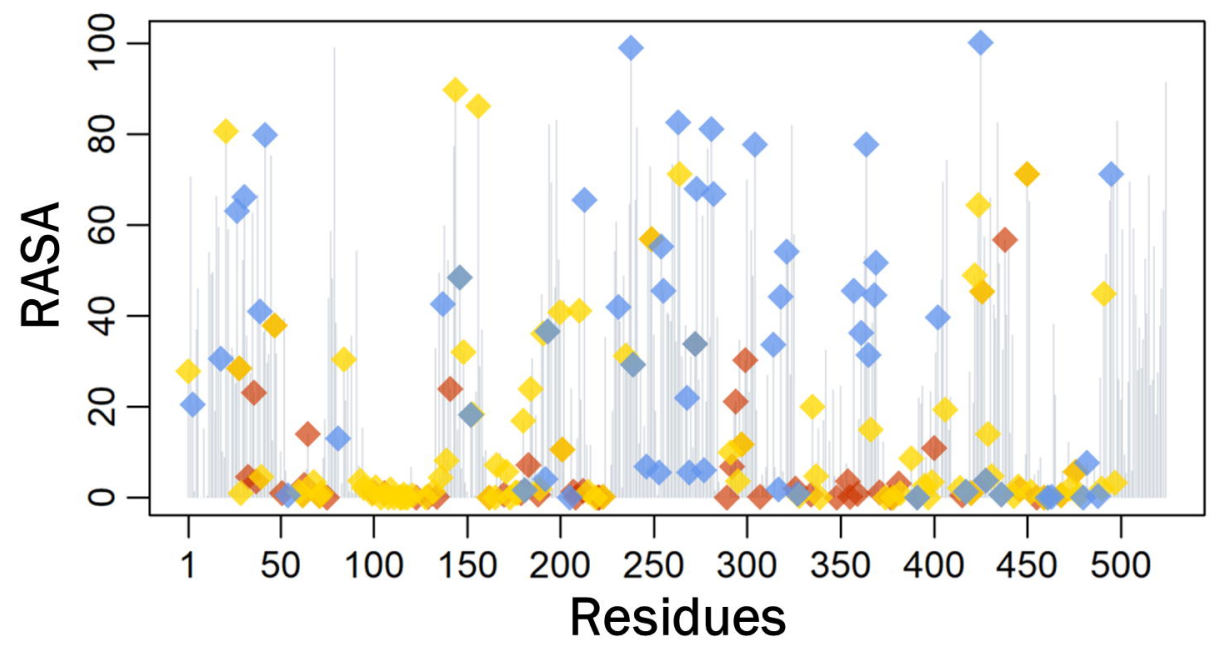

B

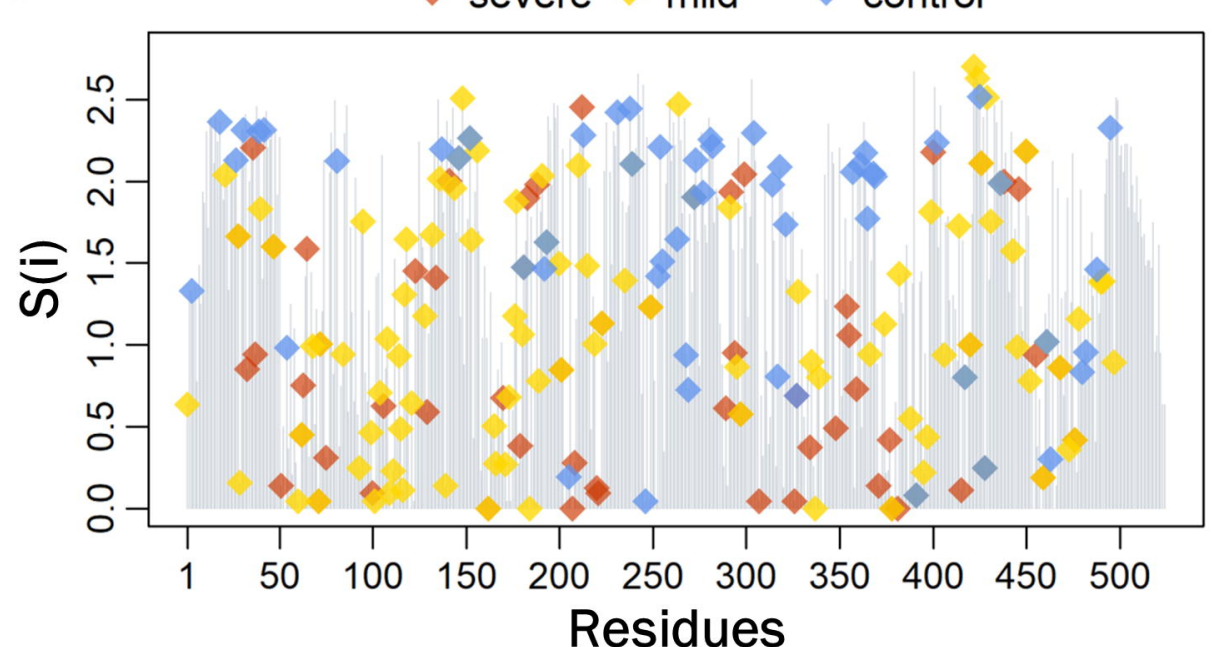

C

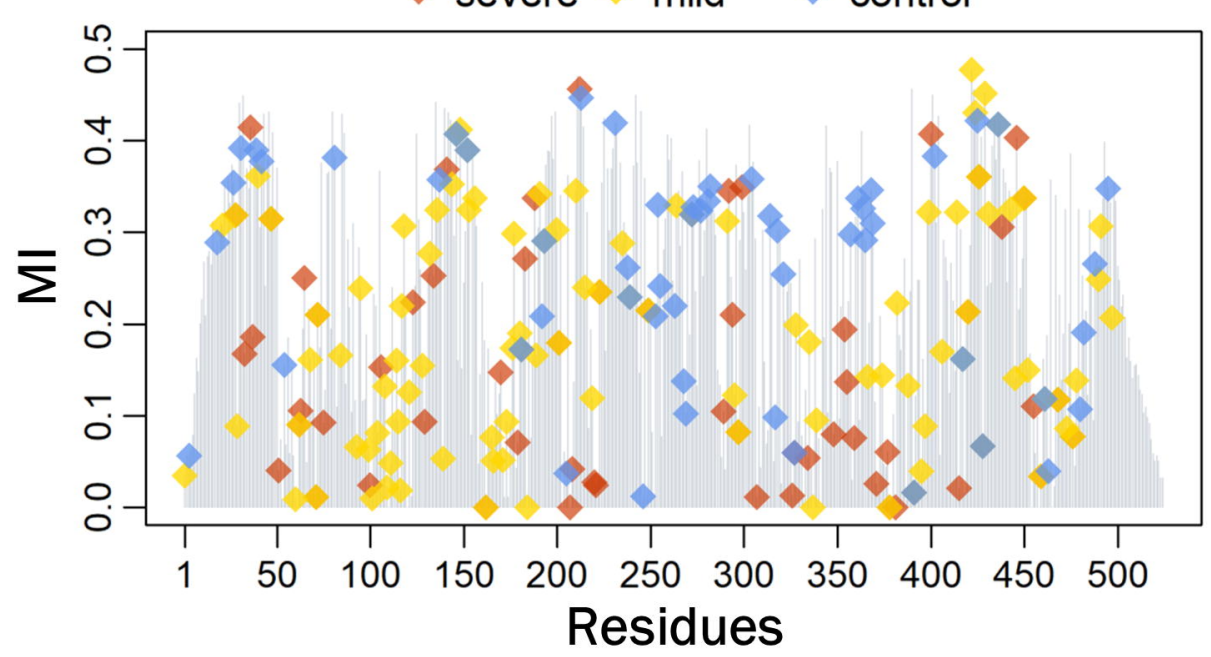

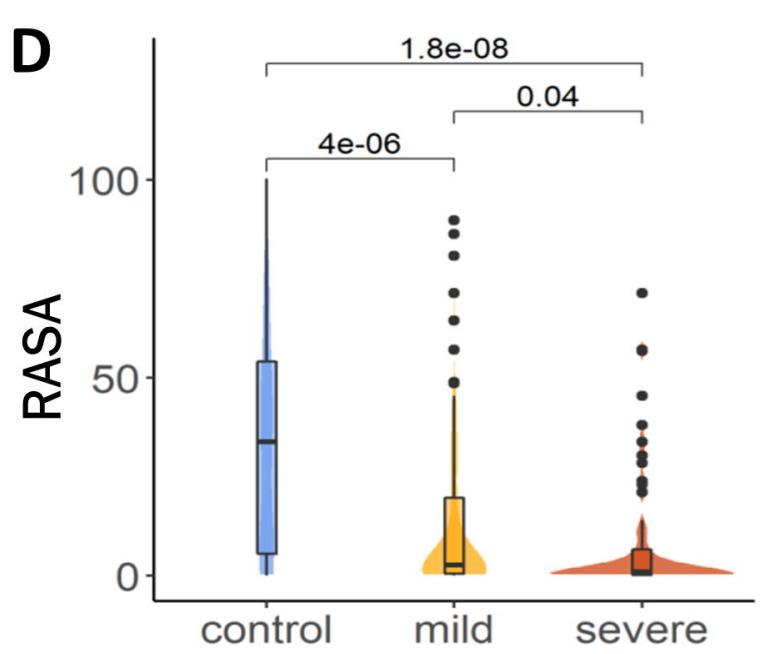

E
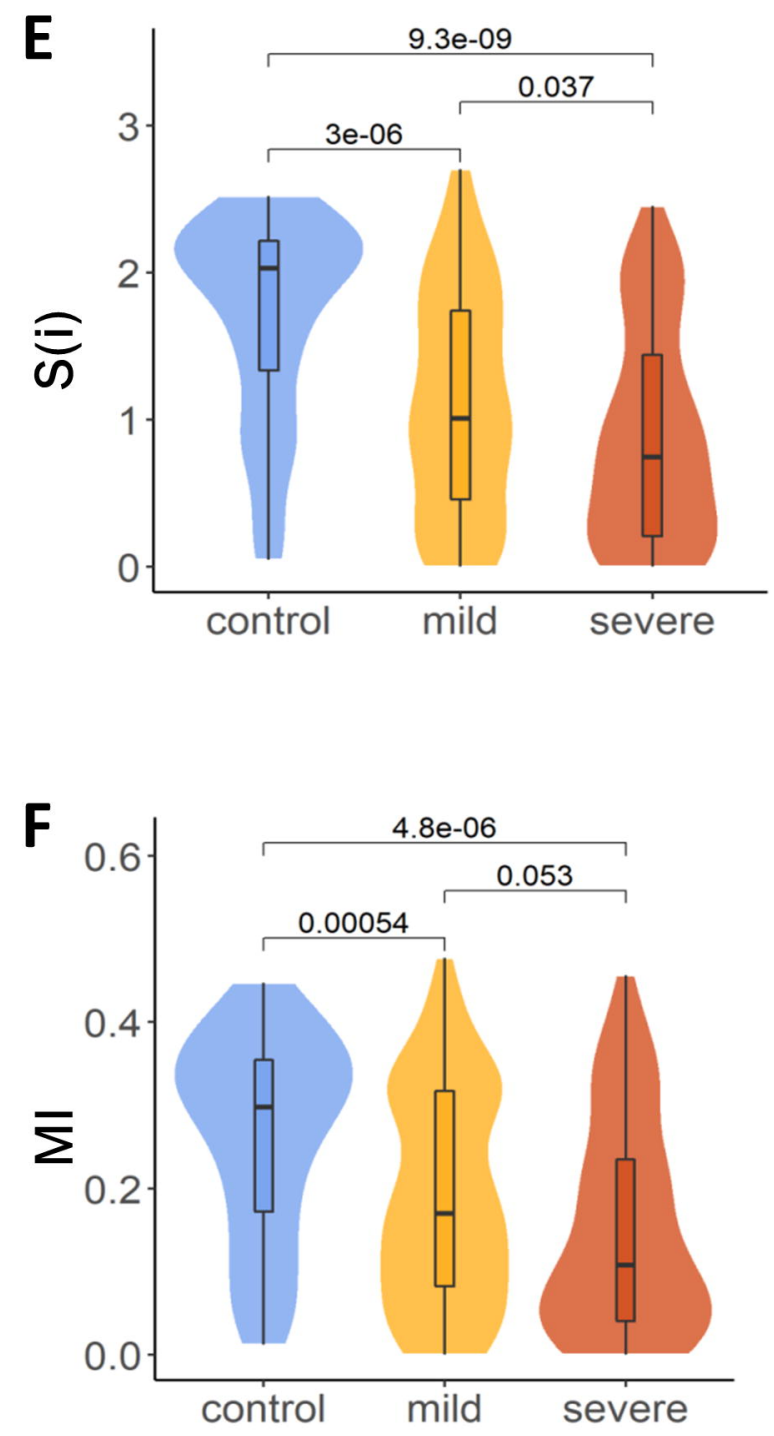

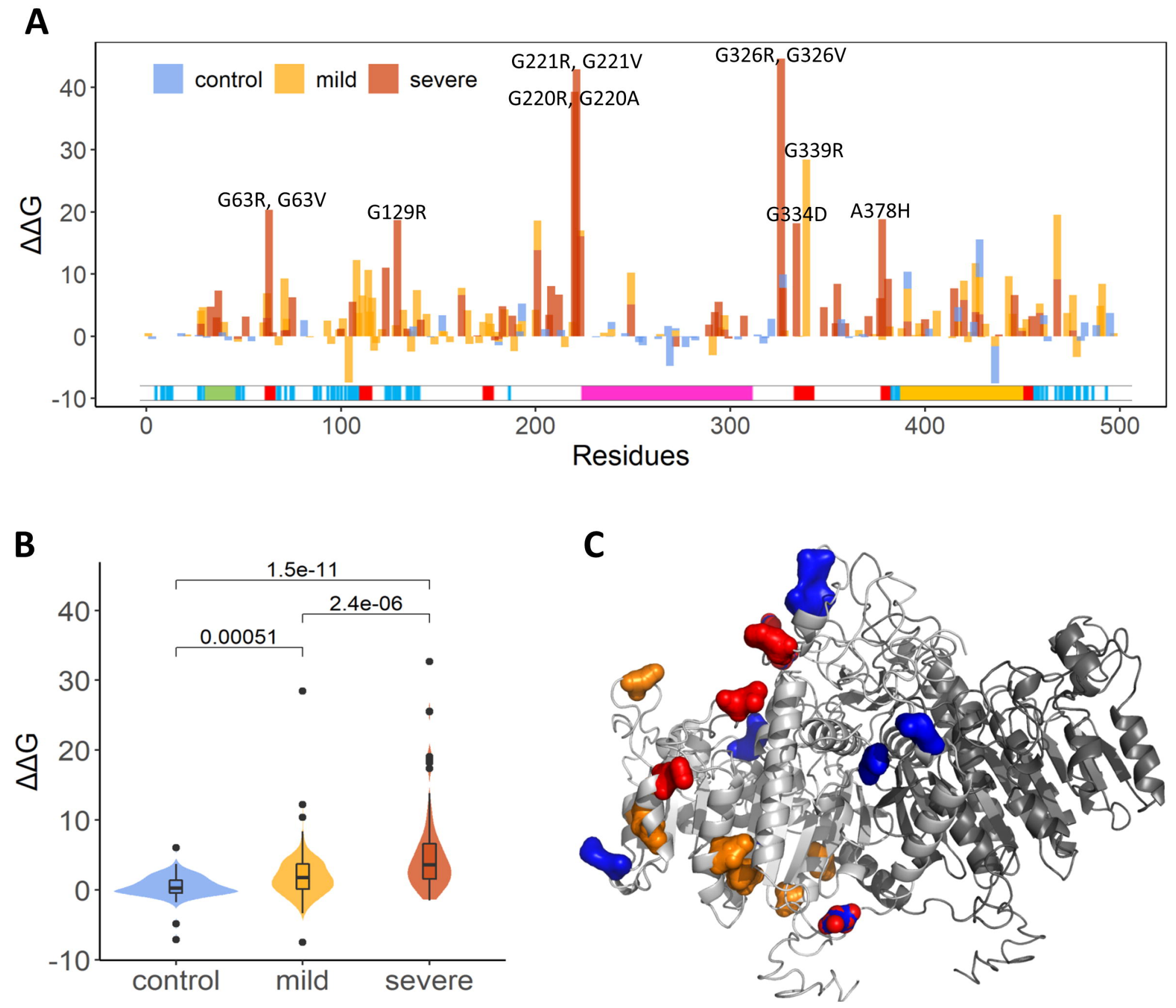


\section{A}

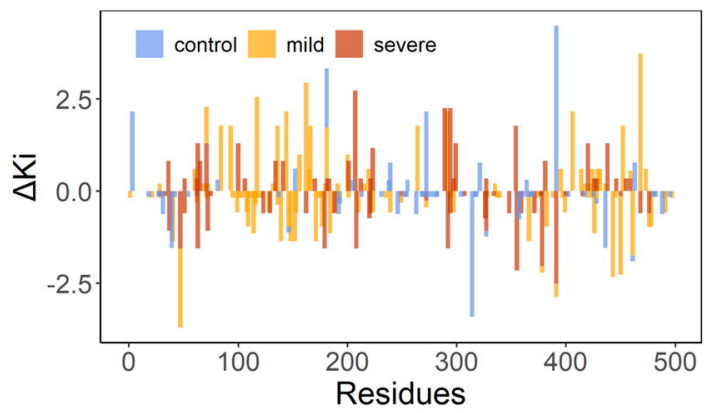

D

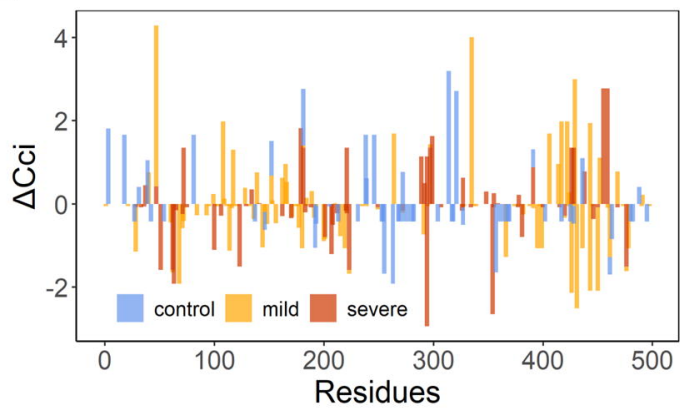

\section{G}

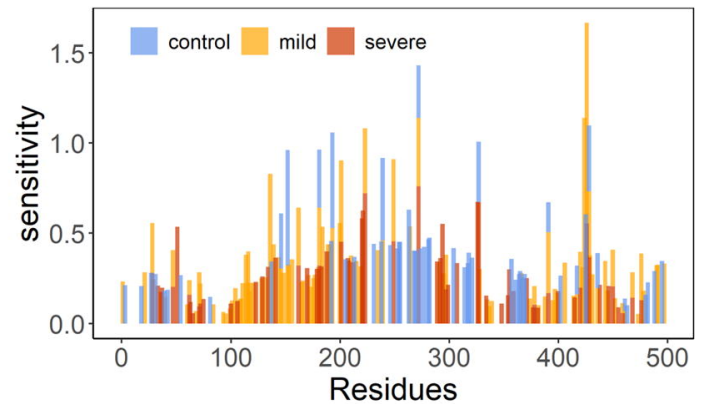

B

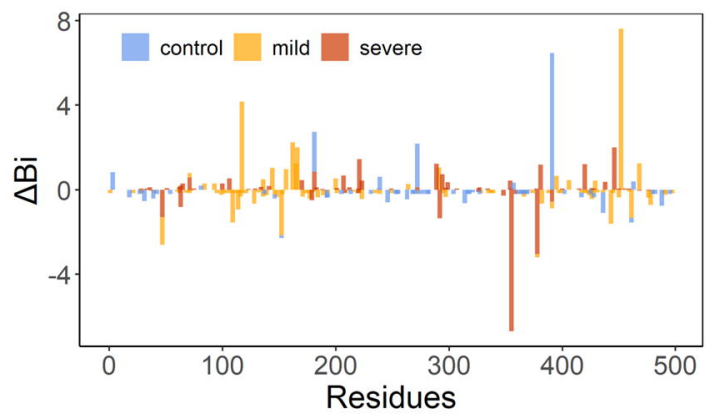

E

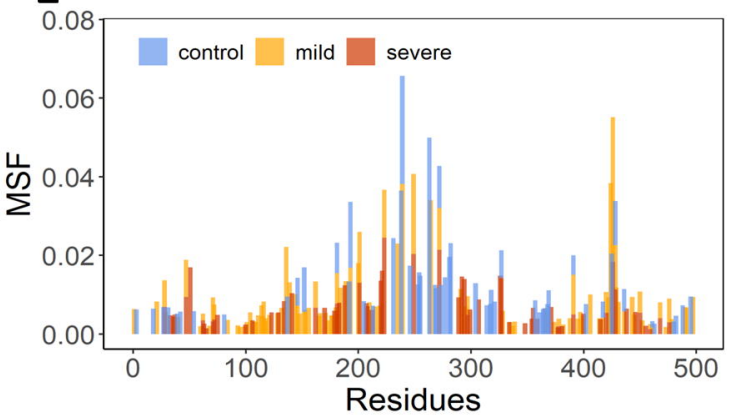

H

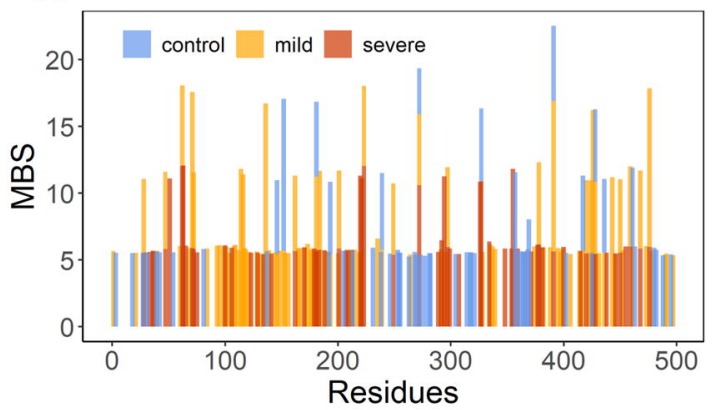

\section{C}

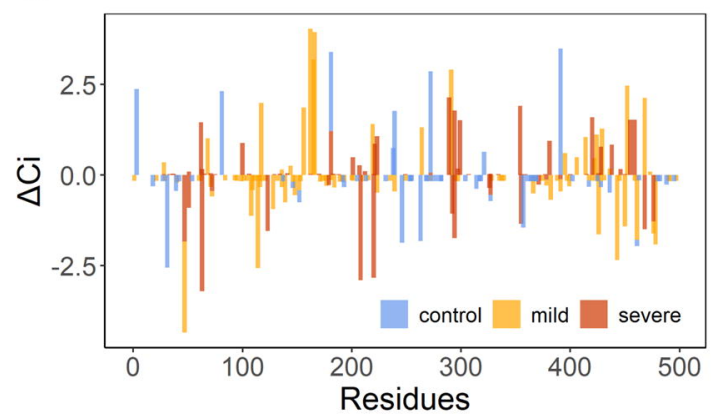

F

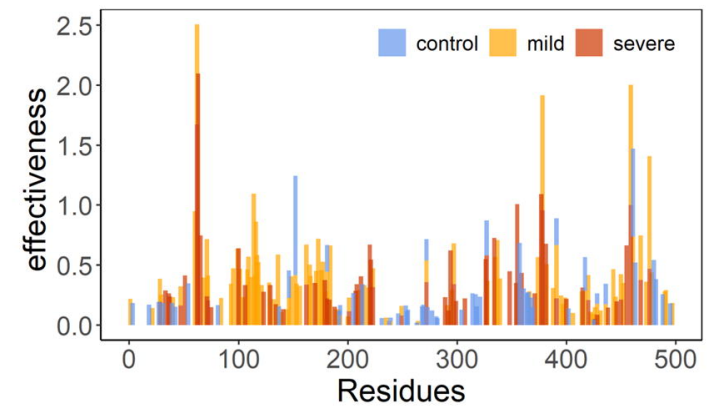

\section{I}

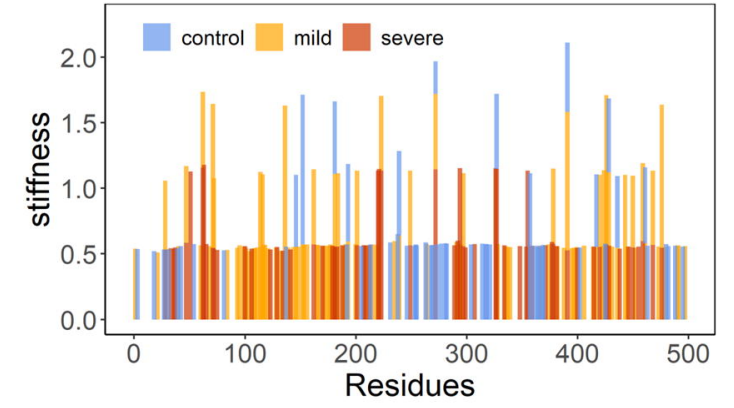


A
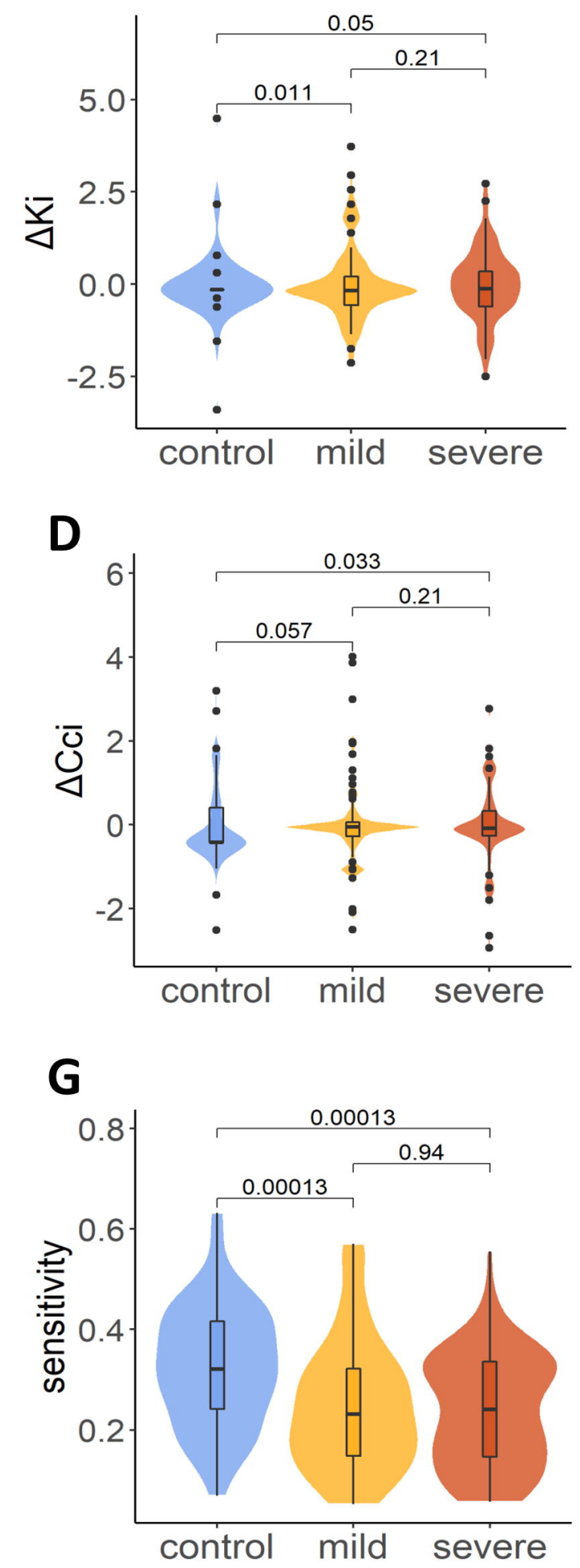

B

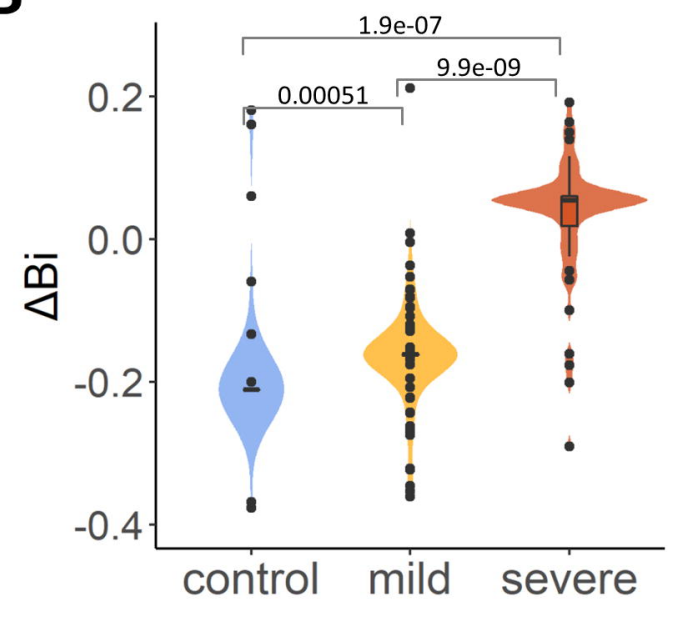

E

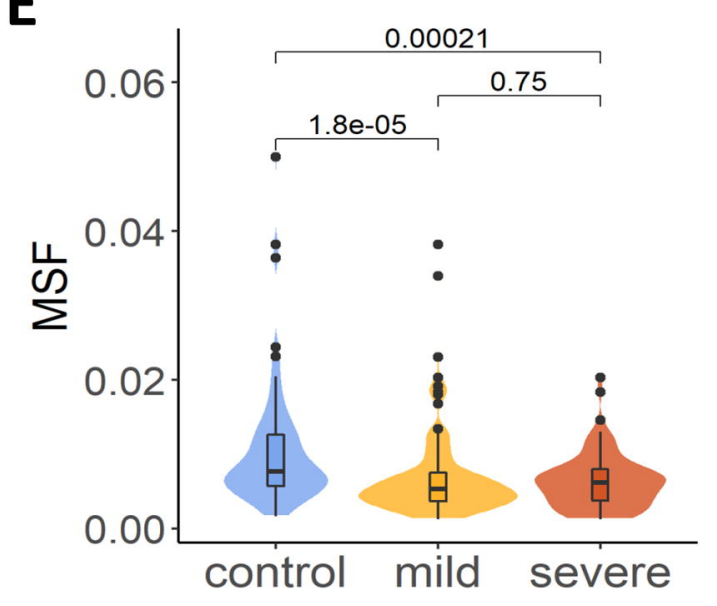

H

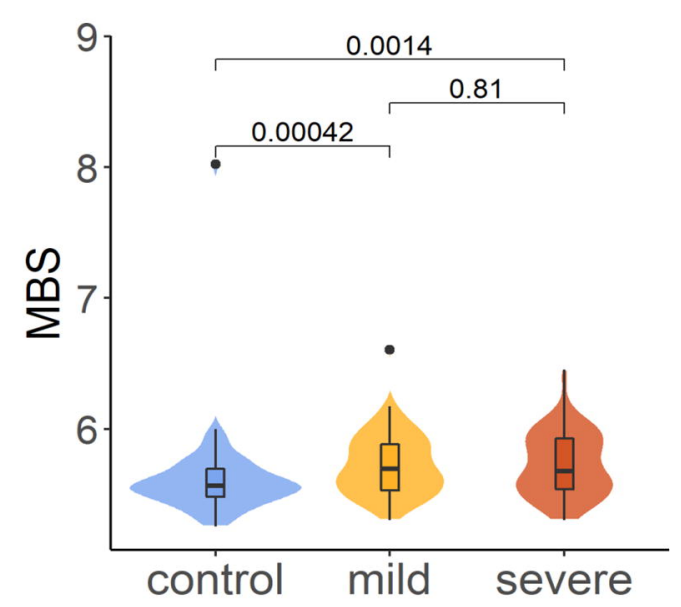

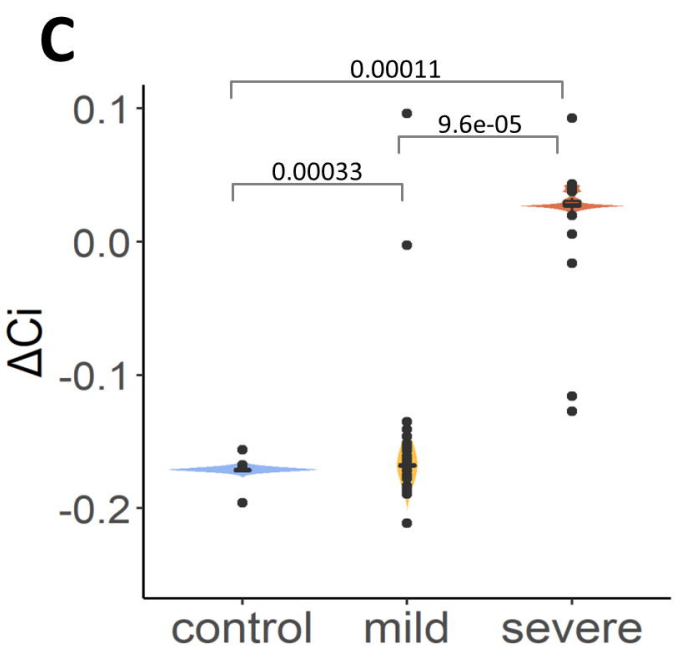
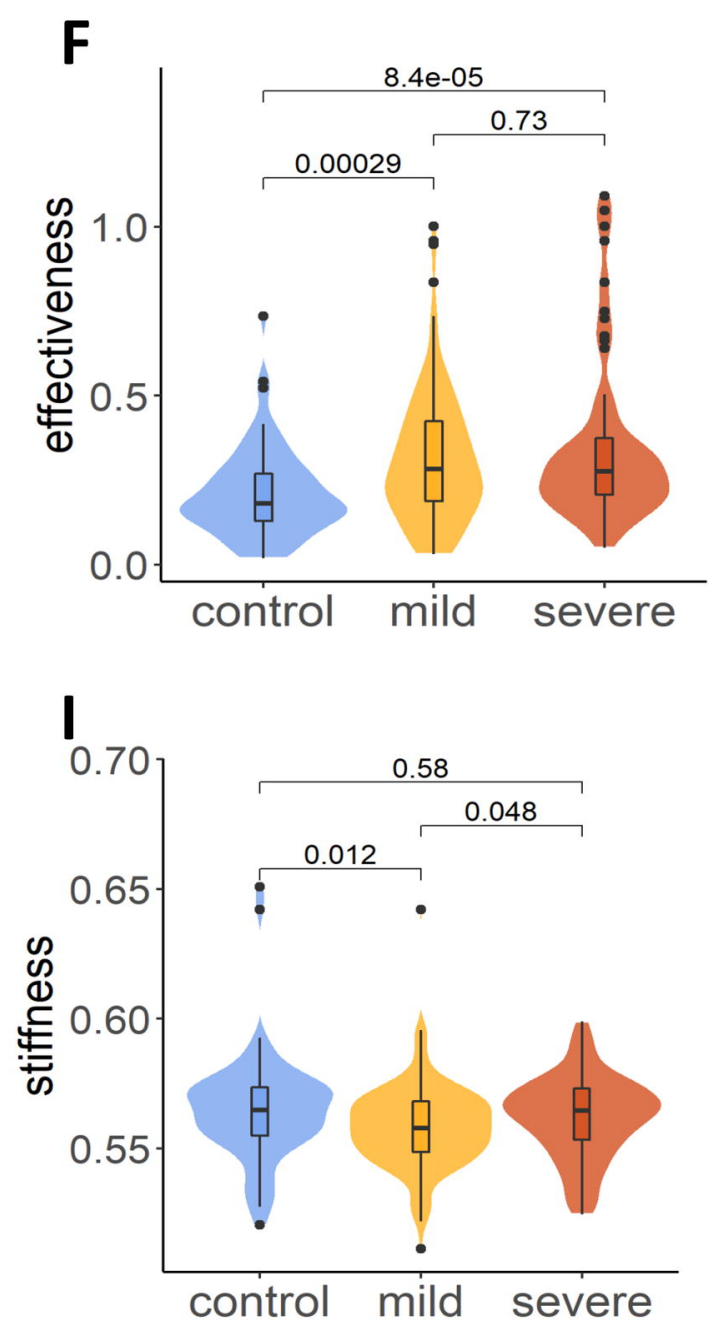


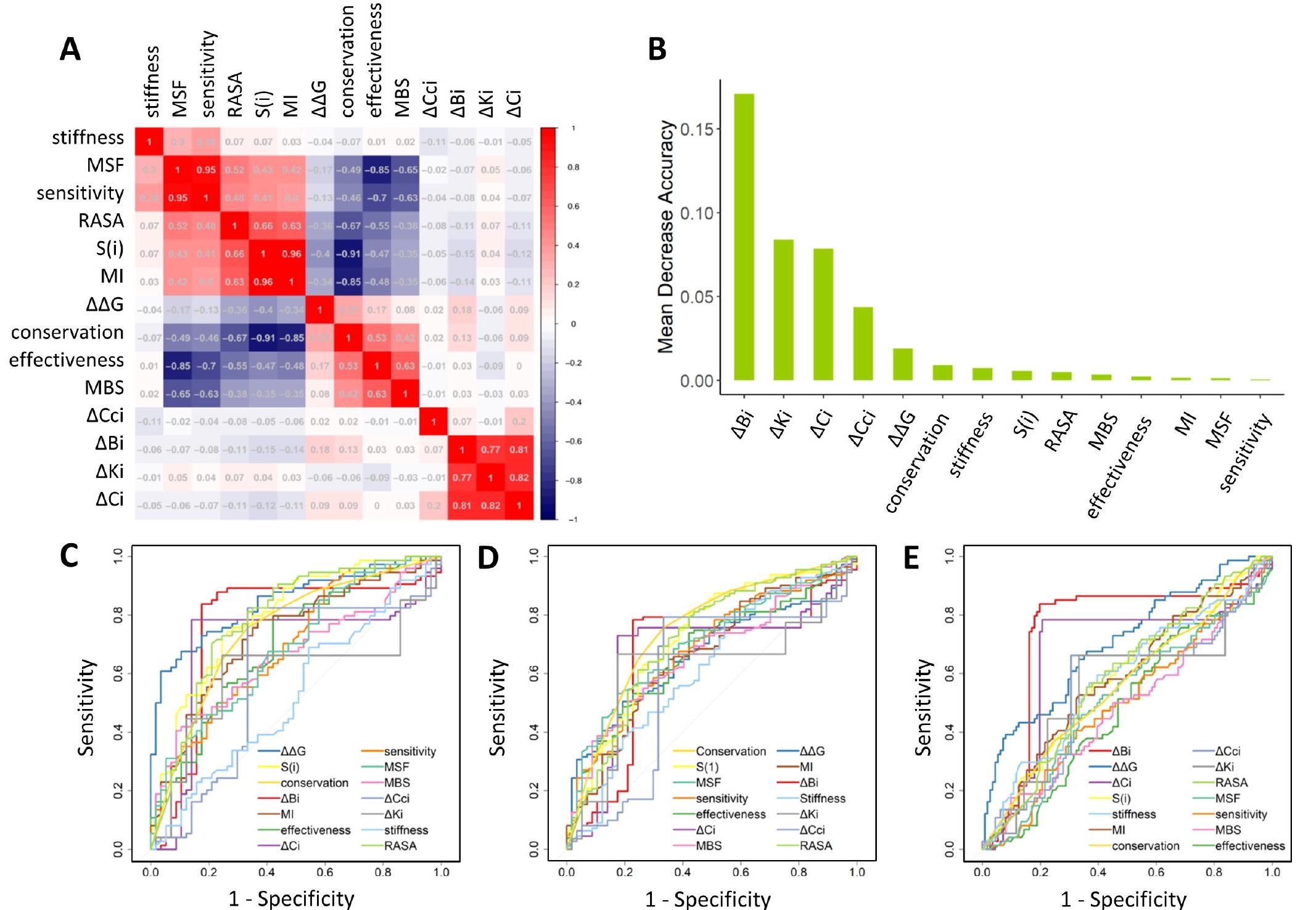




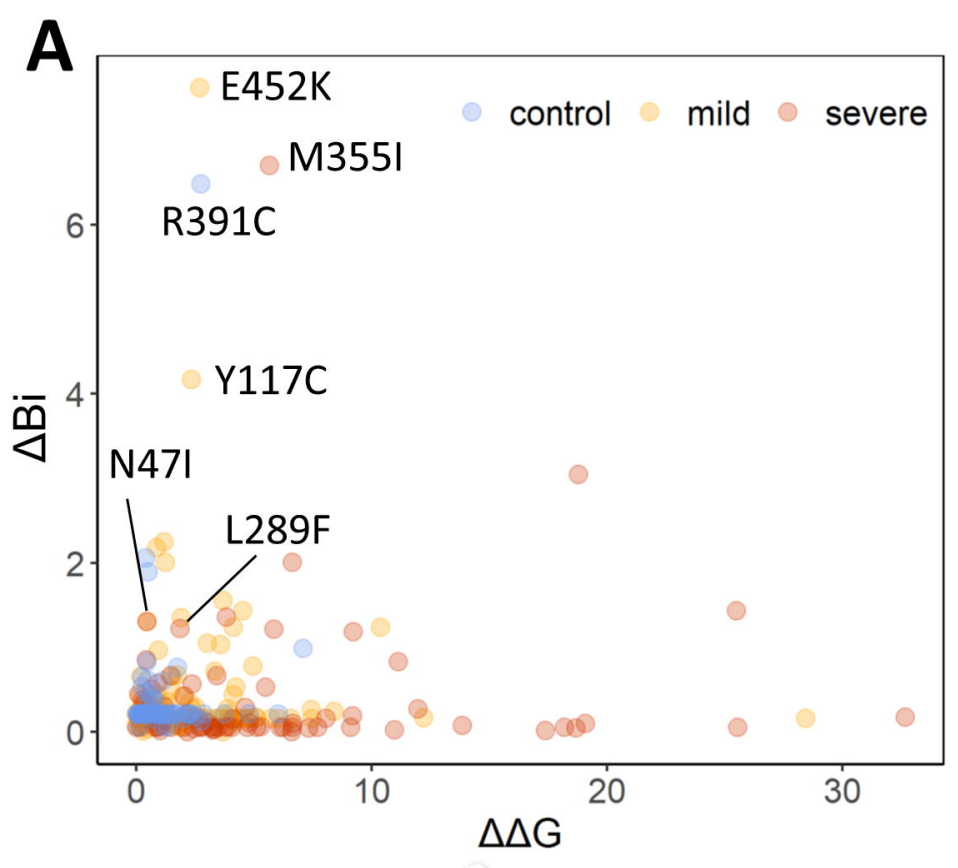

C

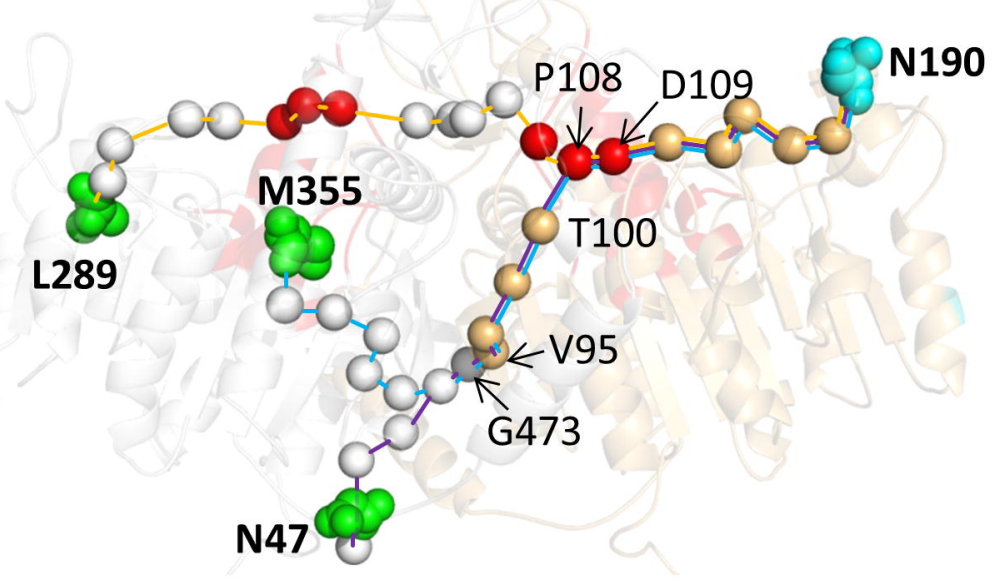

E

WT

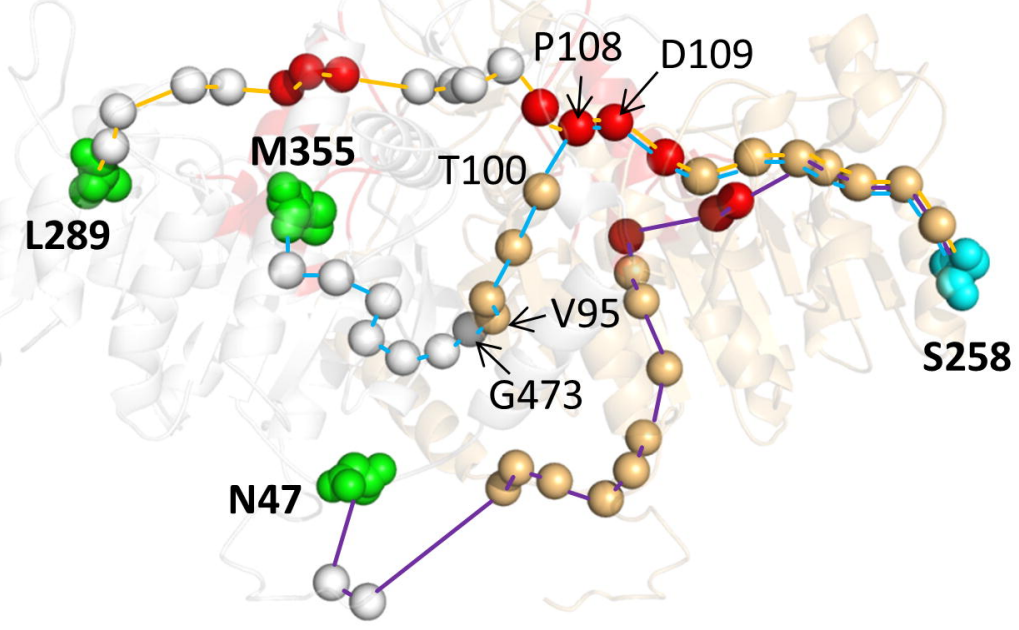

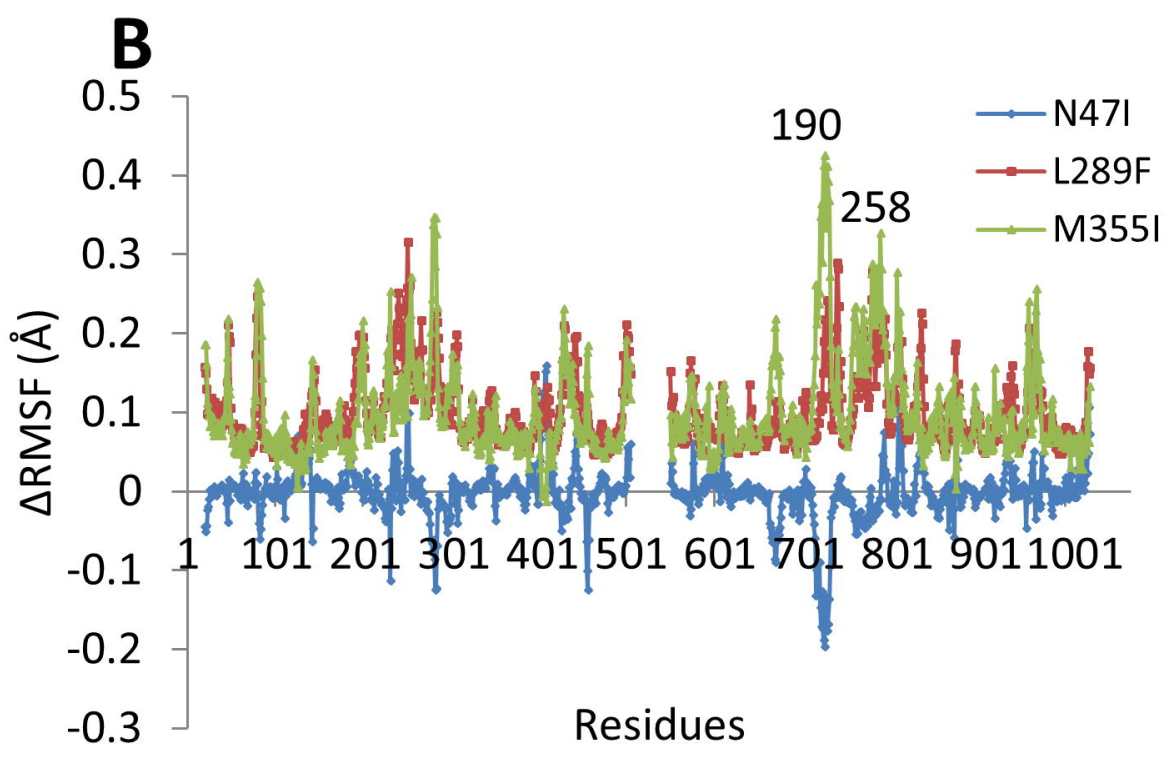

D Mutants

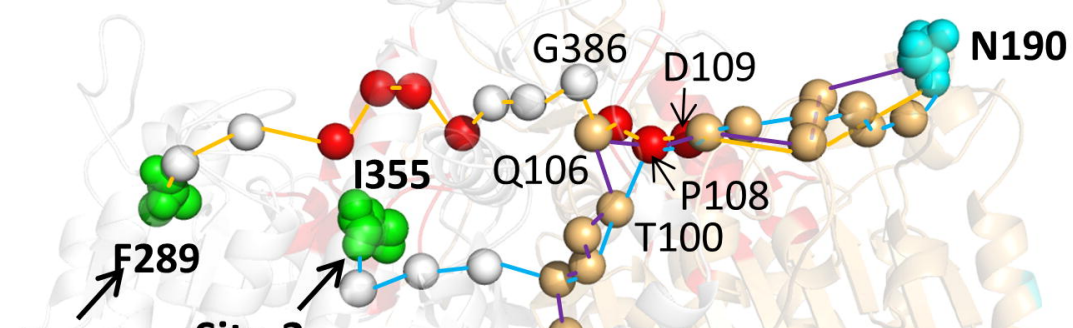

Site 2 Site 3

F

\section{Mutants}

Site 2

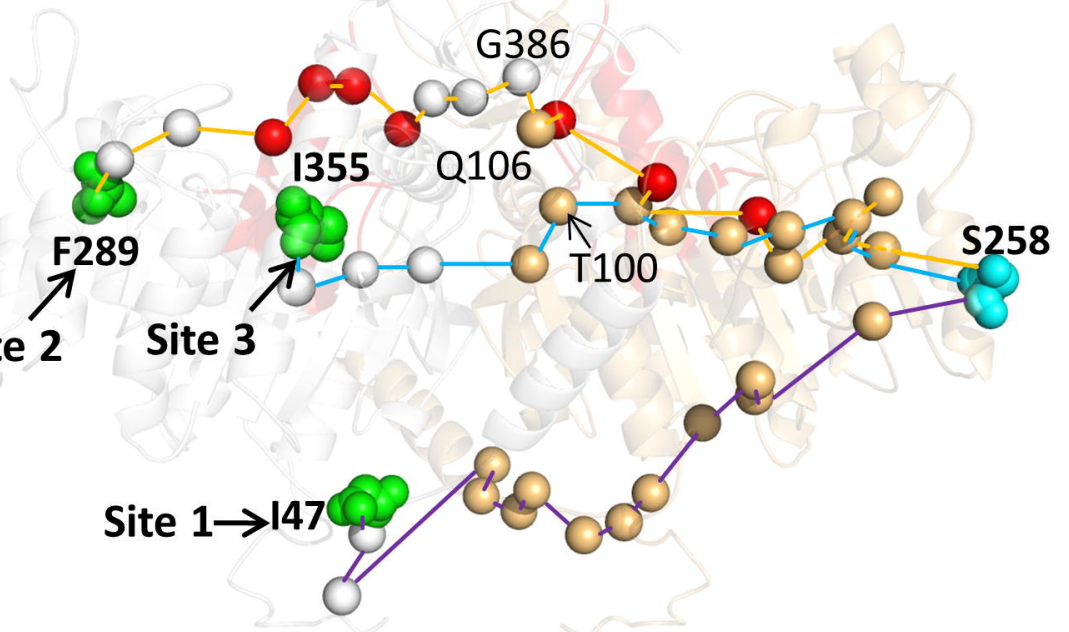


A

$$
\text { singens }
$$

2.

ร5?

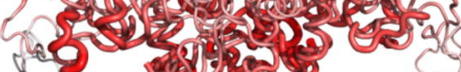

9 \%

(2)

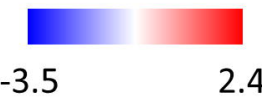

E

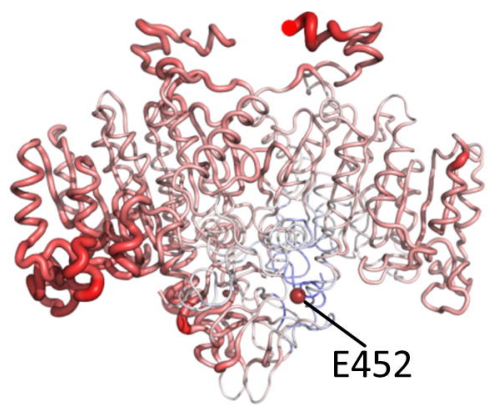

$-1.9$
B

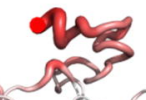
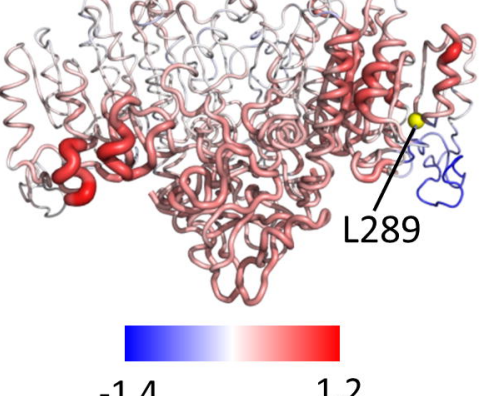

1.2
स्रे
C
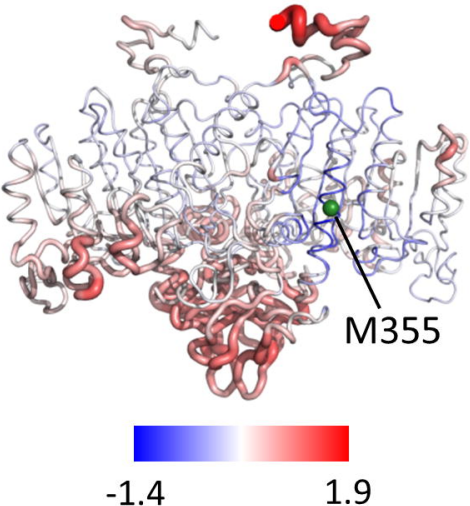

D

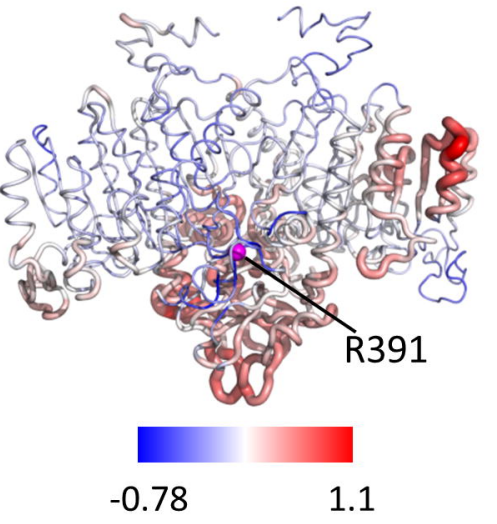

$\mathbf{F}$

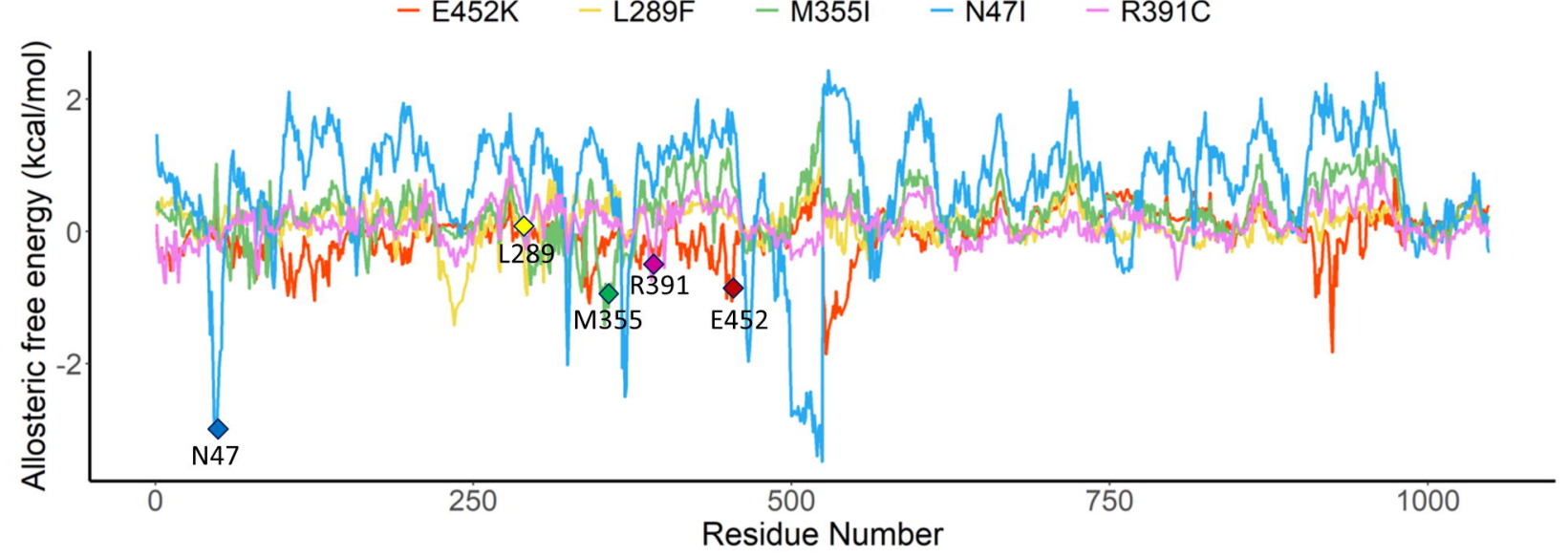

